\title{
How can clinicians choose between conflicting and discordant systematic reviews? A replication study of the Jadad algorithm
}

Carole Lunny ( $\square$ carole.lunny@ubc.ca)

St Michael's Hospital, Unity Health Toronto

Sai Surabi Thirugnanasampanthar

Epidemiology Division and Institute for Health Policy, Management, and Evaluation, Dalla Lana School of Public Health, University of Toronto

Salmaan Kanji

Ottawa Hospital Research Institute

Nicola Ferri

Division of Occupational Medicine, IRCCS Azienda Ospedaliero-Universitaria di Bologna

Dawid Pieper

Faculty of Health Sciences Brandenburg, Brandenburg Medical School Theodor Fontane, Institute for Health Services and Health System Research

Sera Whitelaw

Faculty of Medicine and Health Sciences, McGill University

Sara Tasnim

Cochrane Hypertension Review Group, University of British Columbia

Emma K Reid

Nova Scotia Health Authority

Jia He Zhang

Faculty of Science, University of British Columbia

Banveer Kalkat

Faculty of Pharmaceutical Sciences, University of British Columbia

Yuan Chi

Beijing Yealth Technology Co., Ltd

Reema Abdoulrezzak

Faculty of Pharmaceutical Sciences, University of British Columbia

Di Wen Zheng

Faculty of Pharmaceutical Sciences, University of British Columbia

Lindy R.S. Pangka

Faculty of Pharmaceutical Sciences, University of British Columbia

Xin Ran Wang

Faculty of Pharmaceutical Sciences, University of British Columbia

Parisa Safavi Safavi

Faculty of Pharmaceutical Sciences, University of British Columbia

Anmol Sooch

Faculty of Pharmaceutical Sciences, University of British Columbia

Kevin T. Kang

Faculty of Pharmaceutical Sciences, University of British Columbia

Andrea C Tricco

Epidemiology Division and Institute for Health Policy, Management, and Evaluation, Dalla Lana School of Public Health, University of Toronto

Harrison Nelson

Faculty of Health Sciences, Queen's University

\section{Research Article}

Keywords: Discordance, overviews of reviews, overlapping, systematic reviews, meta-analyses, conflicting, discordant, agreement, concordant, replication, knowledge synthesis, evidence synthesis

Posted Date: February 28th, 2022

DOI: https://doi.org/10.21203/rs.3.rs-1143357/v3

License: @ (i) This work is licensed under a Creative Commons Attribution 4.0 International License. Read Full License 


\section{Abstract}

Introduction: The exponential growth of published systematic reviews (SRs) presents challenges for clinicians seeking to answer clinical questions. In 1997, an algorithm was created by Jadad et al. to choose the best SR across multiple. Our study aims to replicate author assessments using the Jadad algorithm to determine: (i) if we chose the same SR as the authors; and (ii) if we reach the same results.

Methods: We searched MEDLINE, Epistemonikos, and Cochrane Database of SRs. We included any study using the Jadad algorithm. We used consensus building strategies to operationalise the algorithm and to ensure a consistent approach to interpretation.

Results: We identified 21 studies that used the Jadad algorithm to choose one or more SRs. In 62\% (13/21) of cases, we were unable to replicate the Jadad assessment and ultimately chose a different SR than the authors. Overall, 18 out of the $21(86 \%)$ independent Jadad assessments agreed in direction of the findings despite 13 having chosen a different SR.

Conclusions: Our results suggest that the Jadad algorithm is not reproducible between users as there are no prescriptive instructions about how to operationalise the algorithm. In the absence of a validated algorithm, we recommend that healthcare providers, policy makers, patients and researchers address conflicts between review findings by choosing the SR(s) with meta-analysis of RCTs that most closely resemble their clinical, public health, or policy question, are the most recent, comprehensive (i.e. in terms of number of included RCTs), and at the lowest risk of bias.

\section{Highlights}

- This is the first empirical study to replicate Jadad algorithm assessments to evaluate discordance across systematic reviews

- In $62 \%$ (13/21) of cases, we were unable to replicate the Jadad algorithm assessment and ultimately chose a different systematic review than the authors

- When assessing systematic reviews using the Jadad algorithm, some steps of the Jadad algorithm were vague in description, making it difficult to operationalise, interpret, and use

- The Jadad algorithm has several limitations as it does not account for the last literature search of the systematic review and publication recency of included trials.

- To assess discordance in the absence of an algorithm, we recommend clinicians and policy makers consider relevance (objectives that most closely resemble their clinical question), recency (dates of search), comprehensiveness (most trials), and risk of bias (lowest risk of bias SR) when choosing one systematic review across multiple.

\subsection{Background}

Keeping up with current research for a practicing clinician or policy maker is a monumental task. Global research output is increasing exponentially [1] as is the quantity of published systematic reviews being produced yearly [2-4]. Systematic reviews (SRs) help clinicians navigate complex clinical topics by summarising large numbers of primary studies. Between 2000 and 2019, the number of SRs increased more than 20 -fold, with 80 SRs published per day [5].

The increase in the number of SRs means that overlapping and redundant reviews are increasingly found on the same clinical, public health, or policy question of interest. Bolland et al. found 24 SRs on vitamin D supplements for prevention of bone fractures, and many of these contained conflicting results based on diverse methodological choices and differing included primary studies [6]. When encountering multiple reviews on the same question, clinicians and policy makers may be confused and unable to formulate a conclusive answer to their question [7].

To surmount this challenge, an algorithm was published in 1997 by Jadad et al. [8] to aid healthcare providers and policy makers select the "best evidence" $\mathrm{SR}(\mathrm{s})$ across multiple reviews of randomized controlled trials (RCTs) with meta-analysis. We define discordance as when SRs with identical or nearly identical clinical, public health, or policy questions report different results for the same outcome.

Overviews of reviews (otherwise termed umbrella reviews, meta-reviews, etc.) summarise the results of SRs and can help make sense of potentially conflicting or discordant results [9-12]. However, methods studies aiming to only assess discordance in results across multiple similar SRs have more focused objectives than overviews of reviews. The more focused discordance studies are often called 'reviews of overlapping meta-analyses', 'reviews of discordant SRs', or 'reviews of discordant meta-analyses'. In this paper, we will call them "Discordant Reviews" for clarity and to distinguish them from other types of reviews.

An empirical and systematic mapping study identified formal and informal approaches for dealing with multiple overlapping reviews with discordant results $[10,11]$. One approach was to specify methodological criteria to select a single, most representative SR (e.g., select the highest quality and most comprehensive) [11]. Other identified approaches were to examine and record discordance and use tools (i.e. Jadad algorithm [8]) or decision rules to aid in the selection of one SR $[10,11]$. This systematic mapping study identified only one tool to assess discordance, namely the Jadad algorithm, and given that there are no other options available to assess discordance across SRs, it will continue to be used by researchers. Indeed it still being commonly used today [13-15].

The Jadad algorithm has not been universally adopted and has been inconsistently applied [16-18]. We believe our research is unique as we did not identify any study aiming to replicate the Jadad algorithm. Our study objectives were to: identify Discordant Reviews that used the Jadad algorithm to address discordance amongst conflicting SRs with meta-analysis of RCTs; replicate Jadad assessments done by authors to determine if the same SR(s) would be chosen, and explore reasons for reproducibility or lack thereof.

\subsection{Methods}




\subsection{Study design}

Cochrane SR guidance was followed when performing our study selection and data extraction [19]. Our protocol is registered as a preprint on the Research Square server [20].

\subsection{Search methods}

\subsubsection{Database}

As the basis for our search, we used an existing database of 1218 studies (2000-2020) collated from a bibliometric study [21]. The bibliometric study searched MEDLINE (Ovid), Epistemonikos, and the Cochrane Database of SRs of Interventions (CDSR) between January 1, 2000 and December 30, 2020. All studies included in the database: (a) synthesised the results of SRs, (b) systematically searched for evidence in a minimum of two databases, and (c) conducted their search using a combination of text words and MeSH terms. All included studies also had a full description of methods in the main body of the paper and focused on clinical or public health interventions.

Within this database, we identified Discordant Reviews using the EndNote search function and Boolean logic to identify the following words: overlap* [title/abstract] or discrepan*[title/abstract] or discord*[title/abstract] or concord*[title/abstract] or conflict*[title/abstract] or Jadad [abstract]

\subsubsection{Medline (Ovid) search January to April 2021}

In addition, we completed a more recent search in the first quarter of 2021 in MEDLINE (Ovid) using the following search string: ("SRs".tiab or "metaanalyses".tiab) AND (overlap.tiab or discrepant.tiab or discordant.tiab or difference.tiab or conflicting.tiab or Jadad.ab). This search was conducted on April $18,2021$.

\subsection{Screening}

\subsubsection{Process for screening}

Our screening form was piloted by all authors on 20 studies to ensure high levels of agreement and common definitions of eligibility criteria. Articles were screened as full-text publications independently by two authors. Discrepancies were resolved by consensus, and arbitration by a third reviewer when necessary.

\subsubsection{Stage 1 screening criteria}

We first screened the studies to include all those aiming to assess discordant results across SRs on similar clinical, public health or policy questions. Studies assessing discordance can assess (a) discordant results, or (b) discordant interpretations of the results and conclusions. Both studies examining (a) and (b) were eligible using any approach (e.g. [22-25]). Studies meeting stage 1 criteria continued onto stage 2 screening.

\subsubsection{Stage 2 screening criteria}

In this stage, we selected studies that met the following inclusion criteria:

- Included a minimum of two SRs with a meta-analysis of RCTs, but may have included other study types beyond RCTs; and

- Explicitly used the Jadad algorithm to choose between two or more discordant SRs for the primary outcome.

We included studies in any language and reviewers fluent in other languages used Google translate to aid in screening of non-English studies. Studies were eligible regardless of publication status and publication date.

\subsection{Extraction of the primary intervention and outcome}

As a systematic approach for assessing discordance, we first identified the primary outcome from each Discordant Review. We initially searched for the primary outcome as explicitly defined in the title, abstract, objectives, introduction, or methods sections [26, 27]. If the primary outcome was not identified in any of these sections, we deferred to the first outcome mentioned in the manuscript $[26,27]$.

The primary intervention was selected according to the related to the primary outcome. If multiple interventions were assessed by the primary outcome, we chose the first intervention highlighted in the title or abstract [27]. We then determined which of the included SRs with meta-analysis of RCTs addressed the primary outcome and primary intervention.

The primary intervention and outcome were extracted by two authors independently, and any disagreement was discussed until consensus was reached.

\subsection{Blinding of Jadad results in the Discordant Reviews}

All included manuscripts underwent a blinding process where one reviewer independently deleted content related to Jadad results prior to our independent Jadad assessment. The one reviewer deleted pertinent components of the: abstract, highlights, results of the Jadad assessment, and discussion/conclusions sections, using Adobe Acrobat Pro or the freeware PDFCandy (https://pdfcandy.com). This individual was not involved in the subsequent Jadad assessment. Authors involved in the Jadad assessments were also instructed not to search for and read included Discordant Reviews prior to or during the assessment.

2.6 Achieving consensus instructions on how to do a Jadad assessment 
The Jadad paper provides an algorithm for clinicians to choose across SRs and to identify sources of inconsistency and discordance, including differences in questions, inclusion/exclusion criteria, extracted data, methodological quality assessments, data combining, and statistical analysis methods. Within the Jadad manuscript there is limited detailed guidance regarding the practical operationalisation of the algorithm. As such, authors met virtually to discuss interpretation and application of the algorithm, and to decide upon clear and specific decision rules at each algorithm step. Feedback was solicited and decision rules were adjusted until consensus was achieved. The final Jadad algorithm steps and our decision rules are found in Appendix A, as well as in eight instructional videos located at: https://osf.io/2z7a5/

\subsection{Piloting Jadad assessments}

The consensus instructions underwent pilot testing where further feedback was solicited and adjustments were made. Three Discordant Reviews [6, 26, 27] were used to pilot the Jadad assessments using our instructions. Each two reviewers piloted three assessments using the Jadad algorithm independently and compared to a second reviewer's assessment to identify discrepancies, which were resolved through discussion. Any necessary revisions and clarifications identified through this exercise were noted in our instructions (section 2.8 and Appendix A).

\subsection{Jadad assessment instructions}

Briefly, Step A of the Jadad algorithm involves examining if the included SRs' question match the Discordant Review's question using a PICO framework [8]. If the clinical, public health, or policy questions are not identical, then Step B prompts a user to choose the SR closest to the clinicians' or decision makers' question and no further assessment is necessary. If multiple SRs are found with the same PICO as the Discordant Review, then Step C should be investigated. As we were using Discordant Reviews with the same PICO as their included SRs, we started with Step C in the Jadad algorithm (Fig. 1).

Step C asks whether the same RCTs were included across the SRs (Fig. 1). If the SRs contained the same RCTs, then the assessor moved to Step D and assessed whether the SRs were of the same methodological quality or risk of bias. The AMSTAR (A MeaSurement Tool to Assess systematic Reviews) [22], the updated AMSTAR 2 [23] and outdated Oxman-Guyatt [24] are examples of tools are used to assess methodological quality of SRs, and the ROBIS (Risk of Bias Assessment Tool for Systematic Reviews)( [25] tool is used to assess the risk of bias in SRs. In Step D, we either: (a) extracted the AMSTAR [22], AMSTAR 2 [23], or ROBIS [25] assessments from the Discordant Reviews, or if this was not done, (b) we conducted our own risk of bias assessment using the ROBIS tool [25]. If the included reviews varied in quality, the review of the highest quality was chosen at Step F.

If the SRs were of the same quality/risk of bias, then the next step is Step E - to assess and compare data extraction, clinical heterogeneity, and data synthesis across the reviews. Details about how we assessed this multi-tiered step is found in Appendix A.

If the SRs did not include the same trials, an assessment of the RCTs' eligibility criteria as reported by the SRs was made at Step G. We found eligibility criteria information from the main text in the Discordant Reviews' methods section, or in a table of characteristics. If the information was unavailable in the Discordant Review, two authors extracted the PICO eligibility criteria independently from the included SRs. Any discrepancies were resolved by discussion, and when necessary, with the involvement of a third reviewer.

If the SRs were determined to have sufficiently similar eligibility criteria, Step H prompts the assessor to compare the search strategies and the application of eligibility criteria across SRs to make a selection. If the eligibility criteria are not the same, Step I explores the publication status, quality, language, and availability of data on individual patients across the SRs. We applied multi-tiered hierarchical decision rules for these steps described in detail in Appendix A.

\subsection{Study outcomes}

\subsubsection{Comparing results from our Jadad assessment with the Discordant Review authors' assessment}

We replicated the Jadad assessments and evaluated whether we chose the same SR, whether a Cochrane SR was chosen, and whether we followed the same steps as the Discordant Review authors. We also evaluated the utility, efficiency, and comprehensiveness of the Jadad algorithm, and defined them as:

- Utility: Is the Jadad algorithm easy to use? (section 2.9.2)

- Efficiency: How much time does it take to apply the Jadad algorithm?

- Comprehensiveness: Is the Jadad algorithm missing methods that might explain discordance (e.g., publication recency)?

- Reproducibility: What are the possible reasons for reproducibility or lack thereof?

Our timed Jadad assessments and ease of use ratings started after Steps $\mathrm{C}$ and $\mathrm{G}$ were completed. We therefore can only report our time and utility outcomes to do a partial Jadad assessment (Steps $\mathrm{H}$ and I). Three Discordant Reviews $[6,13,26]$ were used to pilot the Jadad assessments, and were excluded from our assessment of the amount of time it took us to complete Steps $\mathrm{H}$ and I. We also compared our interpretation of how to use the Jadad algorithm with the Discordant Review authors' interpretation.

\subsection{2 "Ease of use" outcome measure}

The Jadad algorithm was assessed for ease of use by each assessor. A colour-coded ranking system was applied (green, yellow, red) based on how easy or difficult the assessment was judged to be for the user. The rating was based on the following rubric:

- The step can be accomplished easily by the reviewer, due to low cognitive load or because it's a recognised method (green).

- The step requires a notable degree of cognitive load by the reviewer but can generally be accomplished with some effort (yellow).

- The step is difficult for the reviewer, due to significant cognitive load or confusion; some reviewers would likely fail or abandon the task at this point (red).

Page 4/22 


\subsubsection{ROBIS assessments}

We assessed all SRs included in the Discordant Reviews for risk of bias using the ROBIS tool [25]. We chose to do this assessment (which is not part of Jadad) to gain knowledge about whether the Discordant Review authors or ourselves chose the SR which was at lowest risk of bias.

\subsection{Data extraction}

Information and data required to complete the Jadad algorithm were first sought directly from the Discordant Reviews, and if not reported, the full texts of the included SRs. The outcomes were extracted from 124 data items outlined in Appendix B. Discordant Review-level and SR-level data were extracted by two authors independently at full-text, and in the case when consensus was not reached, a third author arbitrated. Two reviewers also performed independent extractions of each Discordant Review's interpretation of the Jadad algorithm steps. Any challenges or barriers that authors identified to using the Jadad algorithm was also extracted.

\subsection{Data analysis}

Our analyses were performed (a) descriptively for qualitative data, (b) using frequencies and percentages for categorical data, and (c) using median and interquartile range (IQR) for continuous data.

\subsection{Deviations to our protocol}

Due to the complexity of the Jadad instructions, we made several deviations to our protocol, which are outlined in Appendix C.

\subsection{Results}

\subsection{Search results from the bibliometric study}

We retrieved 16,610 records from the MEDLINE (Ovid), CDSR, and Epistemonikos databases, and 237 records from other sources (Fig. 2). Of the remaining 14,437 records after removal of duplicates, 11,481 were excluded at the title/abstract stage, and 1,738 were excluded at the full text stage. A total of 1,218 articles were included that met our eligibility criteria.

\subsection{Search results from the Jadad replication study}

We updated our search in April 2021, which yielded 1,251 records of which 948 were excluded at the title/abstract stage. We screened 303 full text records, and of these, 24 studies included at least two SRs with meta-analysis of RCTs and used the Jadad algorithm. However, after scrutiny, we excluded another three studies [27-29] from our analysis as they did not choose one or multiple SRs based on the Jadad algorithm and did not follow the Jadad steps. These studies are described separately in Appendix D.

\subsection{Characteristics of Discordant Reviews}

The most common nomenclature for this study type was a 'SR of overlapping meta-analyses', or a 'SR of discrepant meta-analyses'. One study was described by the authors as a 'Systematic review of systematic reviews' in the title and their primary aim was to assess discordance across the SRs, not to synthesize the results of multiple SRs. Other studies self-identified as SRs in the title but they did not collect and analyse primary study data. Instead, the authors of these articles assessed discordance across SRs. Despite the variety in terminology across our included studies, we henceforth call them Discordant Reviews to distinguish them from their constituent SRs.

The 21 Discordant Reviews using the Jadad algorithm were published between 2014 and 2020 (Table 1), except for Poolman 2007 [30]. Overall, more than half of the 21 Discordant Reviews were from China alone; and when counted with the USA, accounted for most of the included Discordant Reviews. Eighteen of the 21 studies ( $86 \%$ ) were done on conditions of the bone and joint, with the rest being on cholecystitis, orthodontically induced white spot lesionslesions, and non-small cell lung cancer.

Within each Discordant Review, the number of included SRs with meta-analysis ranged from 2 to 7, except for Xing 2016, which included 10. The number of authors of the Discordant Reviews ranged from 2 to 10 . 
Table 1

Characteristics of included Discordant Reviews $(n=21)$

\begin{tabular}{|c|c|c|c|c|c|c|c|c|}
\hline $\begin{array}{l}\text { First Author } \\
\text { Year }\end{array}$ & Objective & $\begin{array}{l}\text { Primary } \\
\text { outcome }\end{array}$ & $\begin{array}{l}\text { Primary } \\
\text { intervention }\end{array}$ & $\begin{array}{l}\text { Country of } \\
\text { corresponding } \\
\text { author }\end{array}$ & $\begin{array}{l}\text { Health area } \\
\text { addressed } \\
\text { (ICD-10 } \\
\text { Medical } \\
\text { Classification) }\end{array}$ & $\begin{array}{l}\text { \# Authors } \\
\text { per } \\
\text { Discordant } \\
\text { Review }\end{array}$ & $\begin{array}{l}\text { \# } \\
\text { Systematic } \\
\text { reviews } \\
\text { with meta- } \\
\text { analysis of } \\
\text { RCTs }\end{array}$ & $\begin{array}{l}\text { Discordant } \\
\text { Review } \\
\text { authors' } \\
\text { conclusion }\end{array}$ \\
\hline $\begin{array}{l}\text { Bakdach } \\
2020[13]\end{array}$ & $\begin{array}{l}\text { Appraise evidence } \\
\text { on the } \\
\text { management of } \\
\text { orthodontically } \\
\text { induced white } \\
\text { spot lesions } \\
\text { (OIWSLs) and } \\
\text { choose the best } \\
\text { evidence }\end{array}$ & $\begin{array}{l}\text { Incidence of } \\
\text { lesions }\end{array}$ & $\begin{array}{l}\text { Topical fluoride } \\
\text { toothpaste } \\
\text { and/or brush on } \\
\text { gel [varnish], or } \\
\text { foam }\end{array}$ & Syria & $\begin{array}{l}\text { Caries limited } \\
\text { to enamel } \\
\text { (K02.0) }\end{array}$ & 2 & 3 & $\begin{array}{l}\text { "Topical } \\
\text { fluorides } \\
\text { yielded a 25- } \\
30 \% \text { preventiol } \\
\text { of OIWSLs; } \\
\text { however, their } \\
\text { effect on } \\
\text { reversing } \\
\text { OIWSLs is } \\
\text { unclear." }\end{array}$ \\
\hline $\begin{array}{l}\text { Chalmers } \\
2015 \text { [26] }\end{array}$ & $\begin{array}{l}\text { Critically evaluate } \\
\text { meta-analyses for } \\
\text { arthroscopic } \\
\text { versus open } \\
\text { stabilization } \\
\text { techniques for } \\
\text { shoulder } \\
\text { instability }\end{array}$ & $\begin{array}{l}\text { Recurrent } \\
\text { instability }\end{array}$ & $\begin{array}{l}\text { Open versus } \\
\text { arthroscopic } \\
\text { shoulder } \\
\text { stabilization }\end{array}$ & USA & $\begin{array}{l}\text { Other } \\
\text { instability of } \\
\text { joint (M25.3) }\end{array}$ & 7 & 2 & $\begin{array}{l}\text { "There are no } \\
\text { significant } \\
\text { differences in } \\
\text { failure rates } \\
\text { "[i.e. recurrent } \\
\text { instability]. }\end{array}$ \\
\hline $\begin{array}{l}\text { Bolland } 2014 \\
\text { [6] }\end{array}$ & $\begin{array}{l}\text { Explore why } \\
\text { discordant results } \\
\text { arise across } \\
\text { meta-analyses on } \\
\text { vitamin D } \\
\text { supplements and } \\
\text { fracture }\end{array}$ & Hip fracture & $\begin{array}{l}\text { Vitamin D (+/- } \\
\text { Calcium) }\end{array}$ & New Zealand & $\begin{array}{l}\text { Fracture of } \\
\text { unspecified } \\
\text { body region } \\
\text { (T14.2) }\end{array}$ & 2 & 4 & $\begin{array}{l}\text { "Each of the } 3 \\
\text { meta-analyses } \\
\text { concluded tha' } \\
\text { vitamin D } \\
\text { alone does not } \\
\text { prevent } \\
\text { fractures, } \\
\text { regardless of } \\
\text { dose." }\end{array}$ \\
\hline $\begin{array}{l}\text { Grassi } 2018 \\
\text { [31] }\end{array}$ & $\begin{array}{l}\text { Assess and } \\
\text { analyze current } \\
\text { evidence } \\
\text { regarding patellar } \\
\text { resurfacing and } \\
\text { non-resurfacing in } \\
\text { TKA }\end{array}$ & $\begin{array}{l}\text { Risk of } \\
\text { reoperation }\end{array}$ & $\begin{array}{l}\text { Patellar } \\
\text { resurfacing } \\
\text { versus non- } \\
\text { resurfacing in } \\
\text { total knee } \\
\text { arthroplasty }\end{array}$ & Italy & $\begin{array}{l}\text { Unspecified } \\
\text { complication } \\
\text { of internal } \\
\text { orthopaedic } \\
\text { prosthetic } \\
\text { device, } \\
\text { implant and } \\
\text { graft (T84.9) }\end{array}$ & 8 & 5 & $\begin{array}{l}\text { "Risk of re- } \\
\text { operation was } \\
\text { higher after } \\
\text { non- } \\
\text { resurfacing, } \\
\text { however, when } \\
\text { the authors } \\
\text { considered } \\
\text { only high } \\
\text { quality RCTs, } \\
\text { no differences } \\
\text { were reported.' }\end{array}$ \\
\hline $\begin{array}{l}\text { Erickson } \\
2015 \text { [32] }\end{array}$ & $\begin{array}{l}\text { Compare } \\
\text { nonoperative and } \\
\text { operative } \\
\text { treatment of } \\
\text { patellar } \\
\text { dislocations to } \\
\text { determine the } \\
\text { best available } \\
\text { evidence }\end{array}$ & $\begin{array}{l}\text { Recurrent } \\
\text { patellar } \\
\text { dislocations }\end{array}$ & $\begin{array}{l}\text { Nonoperative } \\
\text { versus operative } \\
\text { treatment }\end{array}$ & USA & $\begin{array}{l}\text { Recurrent } \\
\text { dislocation of } \\
\text { patella } \\
\text { (M22.0) }\end{array}$ & 8 & 2 & $\begin{array}{l}\text { "Operative } \\
\text { treatment of } \\
\text { acute patellar } \\
\text { dislocations } \\
\text { may result in a } \\
\text { lower rate of } \\
\text { recurrent } \\
\text { dislocations } \\
\text { than } \\
\text { nonoperative } \\
\text { treatment." }\end{array}$ \\
\hline $\begin{array}{l}\text { Chen P } 2019 \\
\text { [15] }\end{array}$ & $\begin{array}{l}\text { Determine which } \\
\text { meta-analysis } \\
\text { provides the best } \\
\text { available } \\
\text { evidence for the } \\
\text { use of PRP in the } \\
\text { treatment of knee } \\
\text { osteoarthritis } \\
\text { (KOA) patients }\end{array}$ & $\begin{array}{l}\text { Pain as } \\
\text { measured by } \\
\text { the WOMAC } \\
\text { total score }\end{array}$ & $\begin{array}{l}\text { Platelet-rich } \\
\text { plasma (PRP) } \\
\text { Injection versus } \\
\text { hyaluronic acid } \\
\text { (HA) injection or } \\
\text { placebo }\end{array}$ & China & $\begin{array}{l}\text { Gonarthrosis, } \\
\text { unspecified } \\
\text { (M17.9) }\end{array}$ & 8 & 4 & $\begin{array}{l}\text { "Intra-articular } \\
\text { PRP injection } \\
\text { is more } \\
\text { effective in } \\
\text { terms of pain } \\
\text { relief } \\
\text { and function } \\
\text { improvement } \\
\text { in the } \\
\text { treatment of } \\
\text { KOA patients } \\
\text { than HA and } \\
\text { placebo" }\end{array}$ \\
\hline
\end{tabular}

ACL-R: Anterior cruciate ligament reconstruction; DB: Double-bundle; DR: Double-row; HA: hyaluronic acid; IDD: Intervertebral disc degeneration; IF: Intramedullary fixation; KOA: knee osteoarthritis; MA: meta-analysis; OIWSLs: orthodontically induced white spot lesions; OVCF: Osteoporotic vertebral compression fracture; PKP: Percutaneous balloon kyphoplasty; PF: Plate fixation; PRP: platelet-rich plasma; RCR: rotator cuff repair; ROM: Range of motion; SB: Single-bundle; SR: Single-row 


\begin{tabular}{|c|c|c|c|c|c|c|c|c|}
\hline $\begin{array}{l}\text { First Author } \\
\text { Year }\end{array}$ & Objective & $\begin{array}{l}\text { Primary } \\
\text { outcome }\end{array}$ & $\begin{array}{l}\text { Primary } \\
\text { intervention }\end{array}$ & $\begin{array}{l}\text { Country of } \\
\text { corresponding } \\
\text { author }\end{array}$ & $\begin{array}{l}\text { Health area } \\
\text { addressed } \\
\text { (ICD-10 } \\
\text { Medical } \\
\text { Classification) }\end{array}$ & $\begin{array}{l}\text { \# Authors } \\
\text { per } \\
\text { Discordant } \\
\text { Review }\end{array}$ & $\begin{array}{l}\# \\
\text { Systematic } \\
\text { reviews } \\
\text { with meta- } \\
\text { analysis of } \\
\text { RCTs }\end{array}$ & $\begin{array}{l}\text { Discordant } \\
\text { Review } \\
\text { authors' } \\
\text { conclusion }\end{array}$ \\
\hline $\begin{array}{l}\text { Chen X } 2018 \\
\text { [33] }\end{array}$ & $\begin{array}{l}\text { Identify the } \\
\text { benefits and } \\
\text { disadvantages of } \\
\text { unilateral PKP } \\
\text { versus bilateral } \\
\text { PKP as found in } \\
\text { numerous } \\
\text { discordant meta- } \\
\text { analyses }\end{array}$ & $\begin{array}{l}\text { Pain as } \\
\text { measured by } \\
\text { the short-term } \\
\text { VAS score }\end{array}$ & $\begin{array}{l}\text { Unilateral } \\
\text { percutaneous } \\
\text { balloon } \\
\text { kyphoplasty } \\
\text { (PKP) versus } \\
\text { bilateral PKP }\end{array}$ & China & $\begin{array}{l}\text { Unspecified } \\
\text { osteoporosis } \\
\text { with } \\
\text { pathological } \\
\text { fracture } \\
\text { (M80.9) }\end{array}$ & 10 & 5 & $\begin{array}{l}\text { "Unilateral PKF } \\
\text { required } \\
\text { shorter surgice } \\
\text { time and less } \\
\text { cement } \\
\text { volume, } \\
\text { offering } \\
\text { better pain } \\
\text { relief and } \\
\text { quality of life } \\
\text { at post- } \\
\text { operative shor } \\
\text { term follow- } \\
\text { ups.". }\end{array}$ \\
\hline Xu 2017 [34] & $\begin{array}{l}\text { Interpret and } \\
\text { select amongst } \\
\text { discordant MAs } \\
\text { and providing } \\
\text { surgical } \\
\text { recommendations } \\
\text { for displaced } \\
\text { midshaft clavicle } \\
\text { fracture }\end{array}$ & $\begin{array}{l}\text { Fracture non- } \\
\text { union }\end{array}$ & $\begin{array}{l}\text { Intramedullary } \\
\text { fixation (IF) } \\
\text { versus plate } \\
\text { fixation (PF) for } \\
\text { displaced } \\
\text { midshaft } \\
\text { clavicle fracture }\end{array}$ & China & $\begin{array}{l}\text { Fracture of } \\
\text { clavicle } \\
\text { (S42.0) }\end{array}$ & 7 & 5 & $\begin{array}{l}\text { "The best } \\
\text { available } \\
\text { evidence } \\
\text { indicated that } \\
\text { the differences } \\
\text { between IF anc } \\
\text { PF were not } \\
\text { significant in } \\
\text { terms of } \\
\text { shoulder } \\
\text { function or the } \\
\text { rate of } \\
\text { treatment } \\
\text { failure } \\
\text { [includes } \\
\text { fracture union] }\end{array}$ \\
\hline $\begin{array}{l}\text { Song } 2016 \\
{[35]}\end{array}$ & $\begin{array}{l}\text { Assess } \\
\text { discordant MAs } \\
\text { for treating acute } \\
\text { cholecystitis and } \\
\text { timing of } \\
\text { laparoscopic } \\
\text { cholecystectomy }\end{array}$ & Bile duct injury & $\begin{array}{l}\text { Early (within } 7 \\
\text { days of the } \\
\text { onset of } \\
\text { symptoms) } \\
\text { versus delayed } \\
\text { laparoscopic } \\
\text { cholecystectomy } \\
\text { for acute } \\
\text { cholecystitis }\end{array}$ & China & $\begin{array}{l}\text { Acute } \\
\text { cholecystitis } \\
\text { (K81.0) }\end{array}$ & 6 & 6 & $\begin{array}{l}\text { "The best } \\
\text { available } \\
\text { evidence } \\
\text { indicated a } \\
\text { nonsignificant } \\
\text { difference in } \\
\text { bile duct } \\
\text { injury." }\end{array}$ \\
\hline $\begin{array}{l}\text { Zhao } 2015 \\
\text { [18] }\end{array}$ & $\begin{array}{l}\text { Compare surgical } \\
\text { and conservative } \\
\text { interventions for } \\
\text { the treatment of } \\
\text { displaced } \\
\text { midshaft } \\
\text { clavicular } \\
\text { fractures }\end{array}$ & $\begin{array}{l}\text { Function as } \\
\text { assessed by } \\
\text { the constant } \\
\text { score }\end{array}$ & $\begin{array}{l}\text { Surgical versus } \\
\text { conservative } \\
\text { treatment }\end{array}$ & China & $\begin{array}{l}\text { Fracture of } \\
\text { clavicle } \\
\text { (S42.0) }\end{array}$ & 3 & 3 & $\begin{array}{l}\text { "Surgical } \\
\text { treatment } \\
\text { provides a } \\
\text { lower rate of } \\
\text { overall } \\
\text { treatment } \\
\text { failure and a } \\
\text { better } \\
\text { functional } \\
\text { outcome, but i: } \\
\text { associated } \\
\text { with more } \\
\text { implant-relatec } \\
\text { complications }\end{array}$ \\
\hline $\begin{array}{l}\text { Poolman } \\
2007[30]\end{array}$ & $\begin{array}{l}\text { Evaluate reasons } \\
\text { for differences in } \\
\text { systematic } \\
\text { reviews on bone- } \\
\text { patellar tendon- } \\
\text { bone or } \\
\text { hamstring tendon } \\
\text { autograft }\end{array}$ & $\begin{array}{l}\text { Knee stability } \\
\text { as measured } \\
\text { by pivot shift } \\
\text { test }\end{array}$ & $\begin{array}{l}\text { Hamstring } \\
\text { autograft versus } \\
\text { bone-patellar } \\
\text { tendon-bone } \\
\text { autograft }\end{array}$ & Canada & $\begin{array}{l}\text { Sprain and } \\
\text { strain } \\
\text { involving } \\
\text { (anterior) } \\
\text { (posterior) } \\
\text { cruciate } \\
\text { ligament of } \\
\text { knee (S83.5) }\end{array}$ & 4 & 3 & $\begin{array}{l}\text { "The currently } \\
\text { available best } \\
\text { evidence } \\
\text { suggests that } \\
\text { hamstring } \\
\text { tendon } \\
\text { autografts are } \\
\text { superior for } \\
\text { preventing } \\
\text { anterior knee } \\
\text { pain, and ther€ } \\
\text { is limited } \\
\text { evidence that } \\
\text { bone-patellar } \\
\text { tendon-bone } \\
\text { autografts } \\
\text { provide better } \\
\text { stability." }\end{array}$ \\
\hline
\end{tabular}

ACL-R: Anterior cruciate ligament reconstruction; DB: Double-bundle; DR: Double-row; HA: hyaluronic acid; IDD: Intervertebral disc degeneration; IF: Intramedullary fixation; KOA: knee osteoarthritis; MA: meta-analysis; OIWSLs: orthodontically induced white spot lesions; OVCF: Osteoporotic vertebral compression fracture; PKP: Percutaneous balloon kyphoplasty; PF: Plate fixation; PRP: platelet-rich plasma; RCR: rotator cuff repair; ROM: Range of motion; SB: Single-bundle; SR: Single-row 


\begin{tabular}{|c|c|c|c|c|c|c|c|c|}
\hline $\begin{array}{l}\text { First Author } \\
\text { Year }\end{array}$ & Objective & $\begin{array}{l}\text { Primary } \\
\text { outcome }\end{array}$ & $\begin{array}{l}\text { Primary } \\
\text { intervention }\end{array}$ & $\begin{array}{l}\text { Country of } \\
\text { corresponding } \\
\text { author }\end{array}$ & $\begin{array}{l}\text { Health area } \\
\text { addressed } \\
\text { (ICD-10 } \\
\text { Medical } \\
\text { Classification) }\end{array}$ & $\begin{array}{l}\text { \# Authors } \\
\text { per } \\
\text { Discordant } \\
\text { Review }\end{array}$ & $\begin{array}{l}\text { \# } \\
\text { Systematic } \\
\text { reviews } \\
\text { with meta- } \\
\text { analysis of } \\
\text { RCTs }\end{array}$ & $\begin{array}{l}\text { Discordant } \\
\text { Review } \\
\text { authors' } \\
\text { conclusion }\end{array}$ \\
\hline $\begin{array}{l}\text { Mascarenhas } \\
2014 \text { [17] }\end{array}$ & $\begin{array}{l}\text { Determine } \\
\text { whether double- } \\
\text { row (DR) or } \\
\text { single-row (SR) } \\
\text { rotator cuff repair } \\
\text { provides superior } \\
\text { clinical outcomes } \\
\text { and structural } \\
\text { healing }\end{array}$ & $\begin{array}{l}\text { Function as } \\
\text { measured by } \\
\text { the constant } \\
\text { score }\end{array}$ & $\begin{array}{l}\text { Single row (SR) } \\
\text { versus double } \\
\text { row (DR) rotator } \\
\text { cuff repair (RCR) } \\
\text { techniques }\end{array}$ & USA & $\begin{array}{l}\text { Rotator cuff } \\
\text { syndrome } \\
\text { (M75.1) }\end{array}$ & 7 & 3 & $\begin{array}{l}\text { "It was } \\
\text { determined } \\
\text { that, according } \\
\text { to the current } \\
\text { best available } \\
\text { evidence, } \\
\text { DR RCR } \\
\text { provides } \\
\text { superior } \\
\text { patient } \\
\text { outcomes and } \\
\text { structural } \\
\text { healing when } \\
\text { compared witt } \\
\text { SR RCR." }\end{array}$ \\
\hline $\begin{array}{l}\operatorname{Tan} 2018 \\
{[36]}\end{array}$ & $\begin{array}{l}\text { Compare clinical } \\
\text { safety and } \\
\text { efficacy of } \\
\text { unilateral versus } \\
\text { bilateral PKP for } \\
\text { treating } \\
\text { osteoporotic } \\
\text { vertebral } \\
\text { compression } \\
\text { fracture (OVCF) }\end{array}$ & $\begin{array}{l}\text { Pain as } \\
\text { measured by } \\
\text { the short-term } \\
\text { VAS score }\end{array}$ & $\begin{array}{l}\text { Unilateral versus } \\
\text { bilateral } \\
\text { percutaneous } \\
\text { balloon }\end{array}$ & China & $\begin{array}{l}\text { Unspecified } \\
\text { osteoporosis } \\
\text { with } \\
\text { pathological } \\
\text { fracture } \\
\text { (M80.9) }\end{array}$ & 6 & 6 & $\begin{array}{l}\text { "Compared } \\
\text { with bilateral } \\
\text { PKP, unilateral } \\
\text { PKP produced } \\
\text { a shorter } \\
\text { surgery time, } \\
\text { smaller dosag } \\
\text { of cement, } \\
\text { lower risk of } \\
\text { cement } \\
\text { leakage, and } \\
\text { relieved a } \\
\text { higher degree } \\
\text { of intractable } \\
\text { pain at short- } \\
\text { term follow-up } \\
\text { after surgery." }\end{array}$ \\
\hline $\begin{array}{l}\text { Xing } 2016 \\
\text { [37] }\end{array}$ & $\begin{array}{l}\text { Perform a } \\
\text { systematic review } \\
\text { of overlapping } \\
\text { meta-analyses } \\
\text { investigating the } \\
\text { efficacy and } \\
\text { safety of HA for } \\
\text { KOA }\end{array}$ & $\begin{array}{l}\text { Early and late } \\
\text { knee pain }\end{array}$ & $\begin{array}{l}\text { HA versus } \\
\text { placebo }\end{array}$ & China & $\begin{array}{l}\text { Gonarthrosis, } \\
\text { unspecified } \\
\text { (M17.9) }\end{array}$ & 7 & 10 & $\begin{array}{l}\text { "HA is an } \\
\text { effective } \\
\text { intervention in } \\
\text { treating KOA } \\
\text { without } \\
\text { increased risk } \\
\text { of adverse } \\
\text { events." }\end{array}$ \\
\hline $\begin{array}{l}\text { Mascarenhas } \\
2015 \text { [38] }\end{array}$ & $\begin{array}{l}\text { Compare double- } \\
\text { bundle (DB) or } \\
\text { single-bundle } \\
\text { (SB) anterior } \\
\text { cruciate ligament } \\
\text { reconstruction } \\
\text { (ACL-R) }\end{array}$ & $\begin{array}{l}\text { Knee stability } \\
\text { measurements } \\
\text { by pivot-shift } \\
\text { testing }\end{array}$ & $\begin{array}{l}\text { SB versus DB } \\
\text { anterior cruciate } \\
\text { ligament } \\
\text { reconstruction }\end{array}$ & USA & $\begin{array}{l}\text { Sprain and } \\
\text { strain } \\
\text { involving } \\
\text { (anterior) } \\
\text { (posterior) } \\
\text { cruciate } \\
\text { ligament of } \\
\text { knee (S83.5) }\end{array}$ & 7 & 6 & $\begin{array}{l}\text { "The current } \\
\text { best available } \\
\text { evidence } \\
\text { suggests that } \\
\text { DB ACL-R } \\
\text { provides bette } \\
\text { postoperative } \\
\text { knee stability } \\
\text { than SB ACL-R } \\
\text { whereas } \\
\text { clinical } \\
\text { outcomes and } \\
\text { risk of } \\
\text { graft failure ar } \\
\text { similar } \\
\text { between } \\
\text { techniques." }\end{array}$ \\
\hline $\begin{array}{l}\text { Houck } 2017 \\
\text { [39] }\end{array}$ & $\begin{array}{l}\text { Compare early } \\
\text { versus delayed } \\
\text { motion } \\
\text { rehabilitation } \\
\text { protocols after } \\
\text { rotator cuff repair } \\
\text { to determine } \\
\text { which MAs } \\
\text { provide the best } \\
\text { available } \\
\text { evidence. }\end{array}$ & $\begin{array}{l}\text { Range of } \\
\text { motion } \\
\text { (general ROM; } \\
\text { forward } \\
\text { flexion; } \\
\text { external } \\
\text { rotation) }\end{array}$ & $\begin{array}{l}\text { Early versus } \\
\text { delayed motion } \\
\text { rehabilitation } \\
\text { protocols }\end{array}$ & USA & $\begin{array}{l}\text { Rotator cuff } \\
\text { syndrome } \\
\text { (M75.1) }\end{array}$ & 5 & 5 & $\begin{array}{l}\text { "The current, } \\
\text { best available } \\
\text { evidence } \\
\text { suggests that } \\
\text { early motion } \\
\text { improves ROM } \\
\text { after rotator } \\
\text { cuff repair but } \\
\text { increases the } \\
\text { risk of rotator } \\
\text { cuff retear." }\end{array}$ \\
\hline
\end{tabular}

ACL-R: Anterior cruciate ligament reconstruction; DB: Double-bundle; DR: Double-row; HA: hyaluronic acid; IDD: Intervertebral disc degeneration; IF: Intramedullary fixation; KOA: knee osteoarthritis; MA: meta-analysis; OIWSLs: orthodontically induced white spot lesions; OVCF: Osteoporotic vertebral compression fracture; PKP: Percutaneous balloon kyphoplasty; PF: Plate fixation; PRP: platelet-rich plasma; RCR: rotator cuff repair; ROM: Range of motion; SB: Single-bundle; SR: Single-row 


\begin{tabular}{|c|c|c|c|c|c|c|c|c|}
\hline $\begin{array}{l}\text { First Author } \\
\text { Year }\end{array}$ & Objective & $\begin{array}{l}\text { Primary } \\
\text { outcome }\end{array}$ & $\begin{array}{l}\text { Primary } \\
\text { intervention }\end{array}$ & $\begin{array}{l}\text { Country of } \\
\text { corresponding } \\
\text { author }\end{array}$ & $\begin{array}{l}\text { Health area } \\
\text { addressed } \\
\text { (ICD-10 } \\
\text { Medical } \\
\text { Classification) }\end{array}$ & $\begin{array}{l}\text { \# Authors } \\
\text { per } \\
\text { Discordant } \\
\text { Review }\end{array}$ & $\begin{array}{l}\# \\
\text { Systematic } \\
\text { reviews } \\
\text { with meta- } \\
\text { analysis of } \\
\text { RCTs }\end{array}$ & $\begin{array}{l}\text { Discordant } \\
\text { Review } \\
\text { authors' } \\
\text { conclusion }\end{array}$ \\
\hline \multirow[t]{2}{*}{$\begin{array}{l}\text { Pekala } 2019 \\
{[40]}\end{array}$} & $\begin{array}{l}\text { Present a } \\
\text { comprehensive } \\
\text { review based on } \\
\text { the most up-to- } \\
\text { date MAs on the } \\
\text { association of } \\
\text { Fokl with IDD }\end{array}$ & $\begin{array}{l}\text { Intervertebral } \\
\text { disc } \\
\text { degeneration } \\
\text { (IDD) }\end{array}$ & $\begin{array}{l}\text { Fokl } \\
\text { (rs2228570) } \\
\text { polymorphism }\end{array}$ & Poland & $\begin{array}{l}\text { Intervertebral } \\
\text { disc disorder, } \\
\text { unspecified } \\
\text { (M51.9) }\end{array}$ & 7 & 7 & $\begin{array}{l}\text { "Based on the } \\
\text { results from } \\
\text { studies } \\
\text { published to } \\
\text { date, there is } \\
\text { no } \\
\text { evidence of an } \\
\text { association } \\
\text { between the } \\
\text { Fokl } \\
\text { polymorphism } \\
\text { and }\end{array}$ \\
\hline & & & & & & & & $\begin{array}{l}\text { IDD in the } \\
\text { general } \\
\text { population." }\end{array}$ \\
\hline $\begin{array}{l}\text { Zhiyong } \\
2019 \text { [41] }\end{array}$ & $\begin{array}{l}\text { Select the best } \\
\text { evidence between } \\
\text { unilateral and } \\
\text { bilateral balloon } \\
\text { kyphoplasty for } \\
\text { osteoporotic } \\
\text { vertebral } \\
\text { compression } \\
\text { fractures (OVCFs) }\end{array}$ & $\begin{array}{l}\text { Pain as } \\
\text { measured by } \\
\text { the short term } \\
\text { VAS scores }\end{array}$ & $\begin{array}{l}\text { Unilateral versus } \\
\text { bilateral balloon } \\
\text { kyphoplasty }\end{array}$ & China & $\begin{array}{l}\text { Unspecified } \\
\text { osteoporosis } \\
\text { with } \\
\text { pathological } \\
\text { fracture } \\
\text { (M80.9) }\end{array}$ & 5 & 6 & $\begin{array}{l}\text { "Unilateral } \\
\text { kyphoplasty is } \\
\text { more } \\
\text { advantageous } \\
\text { effective and } \\
\text { safe, compare } \\
\text { to bilateral } \\
\text { kyphoplasty } \\
\text { for the } \\
\text { treatment of } \\
\text { OVCFs." }\end{array}$ \\
\hline Fu 2019 [42] & $\begin{array}{l}\text { Provide } \\
\text { recommendations } \\
\text { for displaced 3- } \\
\text { part and 4-part } \\
\text { fractures of } \\
\text { proximal humerus } \\
\text { based on the best } \\
\text { evidence }\end{array}$ & $\begin{array}{l}\text { Function as } \\
\text { assessed by } \\
\text { the constant } \\
\text { score }\end{array}$ & $\begin{array}{l}\text { Surgical versus } \\
\text { non-surgical } \\
\text { treatment }\end{array}$ & China & $\begin{array}{l}\text { Fracture of } \\
\text { upper end of } \\
\text { humerus } \\
(\mathrm{S} 42.2)\end{array}$ & 4 & 4 & $\begin{array}{l}\text { "No } \\
\text { statistically } \\
\text { significant } \\
\text { differences } \\
\text { were found in } \\
\text { the constant } \\
\text { score between } \\
\text { surgical and } \\
\text { non-surgical } \\
\text { treatments." }\end{array}$ \\
\hline $\begin{array}{l}\text { Zhao } 2015 \\
\text { [43] }\end{array}$ & $\begin{array}{l}\text { Compare } \\
\text { intramedullary } \\
\text { nail and plate } \\
\text { fixation for the } \\
\text { treatment of } \\
\text { humeral shaft } \\
\text { fractures }\end{array}$ & Non-union & $\begin{array}{l}\text { Intramedullary } \\
\text { nail versus plate } \\
\text { fixation }\end{array}$ & China & $\begin{array}{l}\text { Fracture of } \\
\text { shaft of } \\
\text { humerus } \\
\text { (S42.3) }\end{array}$ & 4 & 4 & $\begin{array}{l}\text { "The } \\
\text { differences } \\
\text { between } \\
\text { intramedullary } \\
\text { nail and plate } \\
\text { fixation were } \\
\text { not significant } \\
\text { in fracture } \\
\text { union." }\end{array}$ \\
\hline $\begin{array}{l}\text { Guo } 2018 \\
{[44]}\end{array}$ & $\begin{array}{l}\text { Offer treatment } \\
\text { recommendations } \\
\text { based on current } \\
\text { best evidence of } \\
\text { Shenyi Capsule } \\
\text { plus chemo } \\
\text { versus chemo of } \\
\text { non-small cell } \\
\text { lung cancer }\end{array}$ & $\begin{array}{l}\text { Disease } \\
\text { control rate }\end{array}$ & $\begin{array}{l}\text { Shenyi Capsule } \\
\text { plus chemo } \\
\text { versus chemo } \\
\text { alone }\end{array}$ & China & $\begin{array}{l}\text { Malignant } \\
\text { neoplasm of } \\
\text { unspecified } \\
\text { part of } \\
\text { unspecified } \\
\text { bronchus or } \\
\text { lung (C34.90) }\end{array}$ & 5 & 4 & $\begin{array}{l}\text { "Shenyi } \\
\text { capsule plus } \\
\text { chemo could } \\
\text { increase } \\
\text { incidence of } \\
\text { short-term } \\
\text { efficacy, } \\
\text { improve the } \\
\text { quality of life } \\
\text { and survival } \\
\text { rate in } \\
\text { comparison to } \\
\text { chemotherapy }\end{array}$ \\
\hline
\end{tabular}

\subsection{Replication of Jadad assessments and Jadad steps assessed}

Over the 21 Jadad assessments we conducted, we did not once answer yes to Step C, meaning the SRs included in the 21 Discordant Reviews did not contain the same RCTs.

As all SRs across the 21 Discordant Reviews contained different RCTs, we then progressed to assess Step G (i.e. do SRs contain the same selection criterion?). Of these, 17 Discordant Reviews (81\%) were determined to have the same selection criteria across their included SRs so they, and moved onto to Step H, and four Discordant Reviews (19\%) did not, and therefore moved onto Step I. 
Since the included SRs did not contain the same RCTs, we did not use Step D, E and F as final decision steps to select a SR. Within Step I of the algorithm, we found that no SRs performed an individual patient analysis, and this sub-step was not used in assessing discordance.

3.3.1 Frequency of agreement and disagreement in the selection of the most appropriate systematic review(s) using the Jadad algorithm between ourselves and the Discordant Review authors

Of the 21 Jadad assessments, eight decisions (38\%) on which SR(s) to choose agreed, and 13 (62\%) disagreed (Tables 2 and 3 ). The author groups of 18 Discordant Reviews chose one SR, two groups chose two SRs, and one group chose three SRs. Over 21 Jadad assessments, we chose one SR in 16 instances, two SRs on four occasions, and three SRs once. In four cases, we chose the same SR as the Discordant Review authors, but also chose one additional SR.

Of the 21 Jadad assessments, 19 (90\%) reported the Jadad step they used to make their final SR selection (hereafter called the "final decision step"). Of the 13 Jadad assessments that disagreed, in six instances we used the same final decision step as the Discordant Review authors, and we chose a different step seven times. Of the eight Jadad assessments between ourselves and the Discordant Review authors that agreed, six reported the Jadad final decision step. Of these, we used the same step to make our decision three out of six times. 
Table 2

Agreement and disagreement in choice of systematic review(s) in replicated Jadad assessments

\begin{tabular}{|c|c|c|c|c|c|c|c|c|c|}
\hline $\begin{array}{l}\text { First Author } \\
\text { Year }\end{array}$ & $\begin{array}{l}\text { Jadad } \\
\text { assessment } \\
\text { primary } \\
\text { outcome }\end{array}$ & $\begin{array}{l}\text { Jadad } \\
\text { assessment } \\
\text { primary } \\
\text { intervention }\end{array}$ & $\begin{array}{l}\# \\
\text { MAs } \\
\text { of } \\
\text { RCTs }\end{array}$ & $\begin{array}{l}\text { Discordant } \\
\text { Review } \\
\text { authors' or } \\
\text { our Jadad } \\
\text { assessments }\end{array}$ & $\begin{array}{l}\text { Jadad } \\
\text { final } \\
\text { decision } \\
\text { step }\end{array}$ & $\begin{array}{l}\text { SR(s) } \\
\text { chosen }\end{array}$ & $\begin{array}{l}\text { Tool used by the } \\
\text { Discordant Review } \\
\text { authors to assess } \\
\text { the quality of SRs } \\
\text { (judgment if } \\
\text { AMSTAR used) }\end{array}$ & $\begin{array}{l}\text { Cochrane } \\
\text { or non- } \\
\text { Cochrane } \\
\text { SR }\end{array}$ & $\begin{array}{l}\text { ROBIS } \\
\text { assessment }\end{array}$ \\
\hline \multirow[t]{2}{*}{$\begin{array}{l}\text { Bakdach } \\
2020\end{array}$} & $\begin{array}{l}\text { Incidence of } \\
\text { white spot } \\
\text { lesions }\end{array}$ & $\begin{array}{l}\text { Topical fluoride } \\
\text { toothpaste, } \\
\text { brush on gel } \\
\text { and/or foam }\end{array}$ & 3 & $\begin{array}{l}\text { Discordant } \\
\text { Review } \\
\text { authors }\end{array}$ & Step I & $\begin{array}{l}\text { Sardana } \\
2018\end{array}$ & $\begin{array}{l}\text { AMSTAR-2 } \\
\text { (Moderate quality) }\end{array}$ & $\begin{array}{l}\text { Non- } \\
\text { Cochrane }\end{array}$ & Low risk \\
\hline & & & & Our Choice & Step I & $\begin{array}{l}\text { Tasios } \\
2019 \\
\\
\text { (and } \\
\text { Sardana } \\
\text { 2019) }\end{array}$ & $\begin{array}{l}\text { AMSTAR-2 } \\
\text { (Critically low } \\
\text { quality) }\end{array}$ & $\begin{array}{l}\text { Non- } \\
\text { Cochrane }\end{array}$ & Low risk \\
\hline \multirow[t]{3}{*}{$\begin{array}{l}\text { Chalmers } \\
2015\end{array}$} & $\begin{array}{l}\text { Rate of } \\
\text { recurrence }\end{array}$ & $\begin{array}{l}\text { Arthroscopic } \\
\text { surgery }\end{array}$ & 2 & $\begin{array}{l}\text { Discordant } \\
\text { Review } \\
\text { authors }\end{array}$ & Step I & $\begin{array}{l}\text { Pulavarti } \\
2007\end{array}$ & Oxman-Guyatt & Cochrane & Low risk \\
\hline & & & & Our Choice & Step H & $\begin{array}{l}\text { Lenters } \\
2007\end{array}$ & & $\begin{array}{l}\text { Non- } \\
\text { Cochrane }\end{array}$ & Low risk \\
\hline & & & & & & $\begin{array}{l}\text { (and } \\
\text { Pulavarti } \\
\text { 2007) }\end{array}$ & & & \\
\hline \multirow[t]{2}{*}{ Bolland 2014} & Hip fracture & $\begin{array}{l}\text { Vitamin D +/- } \\
\text { calcium versus } \\
\text { placebo }\end{array}$ & 4 & $\begin{array}{l}\text { Discordant } \\
\text { Review } \\
\text { authors }\end{array}$ & NR & $\begin{array}{l}\text { Avenell } \\
2009\end{array}$ & $\begin{array}{l}\text { AMSTAR } \\
\text { (High quality) }\end{array}$ & Cochrane & High risk \\
\hline & & & & Our Choice & Step H & $\begin{array}{l}\text { Avenell } \\
2009\end{array}$ & & & \\
\hline \multirow[t]{2}{*}{ Grassi 2018} & $\begin{array}{l}\text { Re-operation } \\
\text { rate }\end{array}$ & $\begin{array}{l}\text { Patellar } \\
\text { resurfacing } \\
\text { versus non- } \\
\text { resurfacing }\end{array}$ & 5 & $\begin{array}{l}\text { Discordant } \\
\text { Review } \\
\text { authors }\end{array}$ & Step I & He 2011 & $\begin{array}{l}\text { AMSTAR } \\
\text { (High quality) }\end{array}$ & $\begin{array}{l}\text { Non- } \\
\text { Cochrane }\end{array}$ & Low risk \\
\hline & & & & Our Choice & Step H & He 2011 & & & \\
\hline \multirow[t]{2}{*}{ Erikson 2015} & $\begin{array}{l}\text { Patellar } \\
\text { instability }\end{array}$ & $\begin{array}{l}\text { Operative versus } \\
\text { non-operative } \\
\text { treatment }\end{array}$ & 2 & $\begin{array}{l}\text { Discordant } \\
\text { Review } \\
\text { authors }\end{array}$ & NR & Hing 2011 & Oxman-Guyatt & Cochrane & Low risk \\
\hline & & & & Our Choice & Step H & Hing 2011 & & & \\
\hline \multirow[t]{2}{*}{ Chen 2019} & $\begin{array}{l}\text { Pain using } \\
\text { WOMAC } \\
\text { total score }\end{array}$ & PRP injection & 4 & $\begin{array}{l}\text { Discordant } \\
\text { Review } \\
\text { authors }\end{array}$ & Step I & Shen 2017 & Oxman-Guyatt & $\begin{array}{l}\text { Non- } \\
\text { Cochrane }\end{array}$ & Low risk \\
\hline & & & & Our Choice & Step H & $\begin{array}{l}\text { Dai et al } \\
2017\end{array}$ & & $\begin{array}{l}\text { Non- } \\
\text { Cochrane }\end{array}$ & Low risk \\
\hline \multirow[t]{3}{*}{ Song 2016} & $\begin{array}{l}\text { Bile duct } \\
\text { injury }\end{array}$ & $\begin{array}{l}\text { Early versus } \\
\text { delayed } \\
\text { laparoscopic } \\
\text { cholecystectomy }\end{array}$ & 6 & $\begin{array}{l}\text { Discordant } \\
\text { Review } \\
\text { authors }\end{array}$ & Step H & Cao 2015 & $\begin{array}{l}\text { AMSTAR } \\
\text { (High quality) }\end{array}$ & $\begin{array}{l}\text { Non- } \\
\text { Cochrane }\end{array}$ & High risk \\
\hline & & & & $\begin{array}{l}\text { Discordant } \\
\text { Review } \\
\text { authors }\end{array}$ & Step H & Wu 2015 & (High quality) & $\begin{array}{l}\text { Non- } \\
\text { Cochrane }\end{array}$ & Low risk \\
\hline & & & & Our Choice & Step H & $\begin{array}{l}\text { Gurusamy } \\
2013\end{array}$ & (Highest quality) & Cochrane & Low risk \\
\hline \multirow[t]{2}{*}{ Chen 2018} & $\begin{array}{l}\text { Short term } \\
\text { VAS }\end{array}$ & $\begin{array}{l}\text { Unilateral PKP } \\
\text { versus bilateral } \\
\text { PKP }\end{array}$ & 5 & $\begin{array}{l}\text { Discordant } \\
\text { Review } \\
\text { authors }\end{array}$ & Step I & Feng 2015 & $\begin{array}{l}\text { AMSTAR } \\
\text { (High quality) }\end{array}$ & $\begin{array}{l}\text { Non- } \\
\text { Cochrane }\end{array}$ & High risk \\
\hline & & & & Our Choice & Step I & Feng 2015 & (High quality) & & \\
\hline Xu 2017 & Non-union & $\begin{array}{l}\text { Intramedullary } \\
\text { fixation versus } \\
\text { plate fixation }\end{array}$ & 5 & $\begin{array}{l}\text { Discordant } \\
\text { Review } \\
\text { authors }\end{array}$ & Step H & $\begin{array}{l}\text { Lenza } \\
2015 \text { and } \\
\text { Hussain } \\
2016\end{array}$ & $\begin{array}{l}\text { AMSTAR } \\
\text { (Highest and High } \\
\text { quality) }\end{array}$ & $\begin{array}{l}\text { Cochrane } \\
\text { and Non- } \\
\text { Cochrane }\end{array}$ & $\begin{array}{l}\text { Low and } \\
\text { High }\end{array}$ \\
\hline
\end{tabular}




\begin{tabular}{|c|c|c|c|c|c|c|c|c|c|}
\hline $\begin{array}{l}\text { First Author } \\
\text { Year }\end{array}$ & $\begin{array}{l}\text { Jadad } \\
\text { assessment } \\
\text { primary } \\
\text { outcome }\end{array}$ & $\begin{array}{l}\text { Jadad } \\
\text { assessment } \\
\text { primary } \\
\text { intervention }\end{array}$ & $\begin{array}{l}\# \\
\text { MAs } \\
\text { of } \\
\text { RCTs }\end{array}$ & $\begin{array}{l}\text { Discordant } \\
\text { Review } \\
\text { authors' or } \\
\text { our Jadad } \\
\text { assessments }\end{array}$ & $\begin{array}{l}\text { Jadad } \\
\text { final } \\
\text { decision } \\
\text { step }\end{array}$ & $\begin{array}{l}\mathrm{SR}(\mathrm{s}) \\
\text { chosen }\end{array}$ & $\begin{array}{l}\text { Tool used by the } \\
\text { Discordant Review } \\
\text { authors to assess } \\
\text { the quality of SRs } \\
\text { (judgment if } \\
\text { AMSTAR used) }\end{array}$ & $\begin{array}{l}\text { Cochrane } \\
\text { or non- } \\
\text { Cochrane } \\
\text { SR }\end{array}$ & $\begin{array}{l}\text { ROBIS } \\
\text { assessment }\end{array}$ \\
\hline & & & & Our Choice & Step H & $\begin{array}{l}\text { Lenza } \\
2015 \text { and } \\
\text { Hussain } \\
2016\end{array}$ & $\begin{array}{l}\text { (Highest and High } \\
\text { quality) }\end{array}$ & & $\begin{array}{l}\text { Low and } \\
\text { High risk }\end{array}$ \\
\hline \multirow[t]{2}{*}{ Zhao 2015a } & $\begin{array}{l}\text { Constant } \\
\text { score }\end{array}$ & $\begin{array}{l}\text { Surgical versus } \\
\text { conservative } \\
\text { treatment }\end{array}$ & 3 & $\begin{array}{l}\text { Discordant } \\
\text { Review } \\
\text { authors }\end{array}$ & Step H & $\begin{array}{l}\text { Lenza } \\
2013\end{array}$ & $\begin{array}{l}\text { AMSTAR } \\
\text { (High quality) }\end{array}$ & Cochrane & Low risk \\
\hline & & & & Our Choice & Step H & $\begin{array}{l}\text { Lenza } \\
2013\end{array}$ & (High quality) & & \\
\hline \multirow[t]{2}{*}{ Tan 2018} & $\begin{array}{l}\text { Short-term } \\
\text { VAS score }\end{array}$ & $\begin{array}{l}\text { Unilateral versus } \\
\text { bilateral } \\
\text { percutaneous } \\
\text { balloon }\end{array}$ & 6 & $\begin{array}{l}\text { Discordant } \\
\text { Review } \\
\text { authors }\end{array}$ & Step H & Feng 2015 & $\begin{array}{l}\text { AMSTAR } \\
\text { (High quality) }\end{array}$ & $\begin{array}{l}\text { Non- } \\
\text { Cochrane }\end{array}$ & Low risk \\
\hline & & & & Our Choice & Step H & Lin 2013 & (High quality) & $\begin{array}{l}\text { Non- } \\
\text { Cochrane }\end{array}$ & High risk \\
\hline \multirow[t]{2}{*}{$\begin{array}{l}\text { Poolman } \\
2007\end{array}$} & Stability & $\begin{array}{l}\text { Hamstring } \\
\text { versus bone- } \\
\text { patellar tendon- } \\
\text { bone autograft }\end{array}$ & 3 & $\begin{array}{l}\text { Discordant } \\
\text { Review } \\
\text { authors }\end{array}$ & Step F & Biau 2006 & Oxman-Guyatt & $\begin{array}{l}\text { Non- } \\
\text { Cochrane }\end{array}$ & Low risk \\
\hline & & & & Our Choice & Step H & Biau 2006 & & & \\
\hline \multirow[t]{2}{*}{$\begin{array}{l}\text { Mascarenhas } \\
2014\end{array}$} & $\begin{array}{l}\text { Constant } \\
\text { score }\end{array}$ & $\begin{array}{l}\text { Single versus } \\
\text { double row } \\
\text { rotator cuff } \\
\text { repair }\end{array}$ & 3 & $\begin{array}{l}\text { Discordant } \\
\text { Review } \\
\text { authors }\end{array}$ & Step I & $\begin{array}{l}\text { Millett } \\
2014\end{array}$ & Oxman-Guyatt & $\begin{array}{l}\text { Non- } \\
\text { Cochrane }\end{array}$ & Low risk \\
\hline & & & & Our Choice & Step H & $\begin{array}{l}\text { Sheibani- } \\
\text { Rad } 2013\end{array}$ & & $\begin{array}{l}\text { Non- } \\
\text { Cochrane }\end{array}$ & High risk \\
\hline \multirow[t]{3}{*}{ Xing 2016} & $\begin{array}{l}\text { Early and } \\
\text { late knee } \\
\text { pain }\end{array}$ & $\begin{array}{l}\text { Hyaluronic acid } \\
\text { versus placebo }\end{array}$ & 10 & $\begin{array}{l}\text { Discordant } \\
\text { Review } \\
\text { authors }\end{array}$ & Step I & $\begin{array}{l}\text { Bellamy } \\
2006\end{array}$ & $\begin{array}{l}\text { AMSTAR } \\
\text { (Highest quality) }\end{array}$ & Cochrane & High risk \\
\hline & & & & Our Choice & Step H & $\begin{array}{l}\text { Richette } \\
2015\end{array}$ & (High quality) & $\begin{array}{l}\text { Non- } \\
\text { Cochrane }\end{array}$ & High risk \\
\hline & & & & & & $\begin{array}{l}\text { (and } \\
\text { Bellamy } \\
\text { 2006) }\end{array}$ & & & \\
\hline \multirow[t]{2}{*}{$\begin{array}{l}\text { Mascarenas } \\
2015\end{array}$} & $\begin{array}{l}\text { Pivot-shift } \\
\text { test score }\end{array}$ & $\begin{array}{l}\text { Single row } \\
\text { versus double } \\
\text { row rotator cuff } \\
\text { repair } \\
\text { techniques }\end{array}$ & 6 & $\begin{array}{l}\text { Discordant } \\
\text { Review } \\
\text { authors }\end{array}$ & Step I & $\begin{array}{l}\text { Li } 2014, \\
\text { van Eck } \\
2012 \text { and } \\
\text { Tiamklang } \\
2012\end{array}$ & Oxman-Guyatt & $\begin{array}{l}\text { Non- } \\
\text { Cochrane, } \\
\text { Cochrane, } \\
\text { and Non- } \\
\text { Cochrane }\end{array}$ & $\begin{array}{l}\text { Low, Low } \\
\text { and Low } \\
\text { risk }\end{array}$ \\
\hline & & & & Our Choice & Step H & $\begin{array}{l}\text { Li } 2014, \\
\text { van Eck } \\
2012 \text { and } \\
\text { Tiamklang } \\
2012\end{array}$ & & & \\
\hline \multirow[t]{2}{*}{ Guo 2018} & $\begin{array}{l}\text { Disease } \\
\text { control }\end{array}$ & $\begin{array}{l}\text { Shenyi capsule } \\
\text { and chemo } \\
\text { versus chemo } \\
\text { alone }\end{array}$ & 4 & $\begin{array}{l}\text { Discordant } \\
\text { Review } \\
\text { authors }\end{array}$ & Step I & Xia 2014 & $\begin{array}{l}\text { AMSTAR } \\
\text { (Moderate quality) }\end{array}$ & $\begin{array}{l}\text { Non- } \\
\text { Cochrane }\end{array}$ & High risk \\
\hline & & & & Our Choice & Step H & Hu 2011 & (Low quality) & $\begin{array}{l}\text { Non- } \\
\text { Cochrane }\end{array}$ & Low risk \\
\hline \multirow[t]{2}{*}{ Houck 2017} & $\begin{array}{l}\text { Range of } \\
\text { motion }\end{array}$ & $\begin{array}{l}\text { Early versus } \\
\text { delayed motion } \\
\text { rehabilitation }\end{array}$ & 5 & $\begin{array}{l}\text { Discordant } \\
\text { Review } \\
\text { authors }\end{array}$ & Step I & $\begin{array}{l}\text { Riboh } \\
2014\end{array}$ & Oxman-Guyatt & $\begin{array}{l}\text { Non- } \\
\text { Cochrane }\end{array}$ & Low risk \\
\hline & & & & Our Choice & Step H & $\begin{array}{l}\text { Chan } \\
2014\end{array}$ & & $\begin{array}{l}\text { Non- } \\
\text { Cochrane }\end{array}$ & High risk \\
\hline Pekala 2019 & $\begin{array}{l}\text { Inter- } \\
\text { vertebral } \\
\text { disc } \\
\text { degeneration }\end{array}$ & $\begin{array}{l}\text { Fokl } \\
\text { polymorphism }\end{array}$ & 7 & $\begin{array}{l}\text { Discordant } \\
\text { Review } \\
\text { authors }\end{array}$ & Step I & $\begin{array}{l}\text { Pabalan } \\
2016\end{array}$ & $\begin{array}{l}\text { AMSTAR } \\
\text { (Moderate quality) }\end{array}$ & $\begin{array}{l}\text { Non- } \\
\text { Cochrane }\end{array}$ & High risk \\
\hline
\end{tabular}

AMSTAR: A MeaSurement Tool to Assess systematic Reviews; Oxman-Guyatt: Oxman-Guyatt quality assessment questionnaire; PKP: Percutaneous balloon kyphoplasty; PRP: platelet-rich plasma; ROBIS: Risk of Bias Assessment Tool for Systematic Reviews. 


\begin{tabular}{|c|c|c|c|c|c|c|c|c|c|}
\hline $\begin{array}{l}\text { First Author } \\
\text { Year }\end{array}$ & $\begin{array}{l}\text { Jadad } \\
\text { assessment } \\
\text { primary } \\
\text { outcome }\end{array}$ & $\begin{array}{l}\text { Jadad } \\
\text { assessment } \\
\text { primary } \\
\text { intervention }\end{array}$ & $\begin{array}{l}\# \\
\text { MAs } \\
\text { of } \\
\text { RCTs }\end{array}$ & $\begin{array}{l}\text { Discordant } \\
\text { Review } \\
\text { authors' or } \\
\text { our Jadad } \\
\text { assessments }\end{array}$ & $\begin{array}{l}\text { Jadad } \\
\text { final } \\
\text { decision } \\
\text { step }\end{array}$ & $\begin{array}{l}\mathrm{SR}(\mathrm{s}) \\
\text { chosen }\end{array}$ & $\begin{array}{l}\text { Tool used by the } \\
\text { Discordant Review } \\
\text { authors to assess } \\
\text { the quality of SRs } \\
\text { (judgment if } \\
\text { AMSTAR used) }\end{array}$ & $\begin{array}{l}\text { Cochrane } \\
\text { or non- } \\
\text { Cochrane } \\
\text { SR }\end{array}$ & $\begin{array}{l}\text { ROBIS } \\
\text { assessment }\end{array}$ \\
\hline & & & & Our Choice & Step I & $\begin{array}{l}\text { Nong } \\
2016\end{array}$ & (Moderate quality) & $\begin{array}{l}\text { Non- } \\
\text { Cochrane }\end{array}$ & Low risk \\
\hline & & & & & & $\begin{array}{l}\text { (and } \\
\text { Pabalan } \\
\text { 2016) }\end{array}$ & & & \\
\hline \multirow[t]{2}{*}{$\begin{array}{l}\text { Zhiyong } \\
2019\end{array}$} & \multirow[t]{2}{*}{$\begin{array}{l}\text { Short term } \\
\text { VAS scores }\end{array}$} & \multirow[t]{2}{*}{$\begin{array}{l}\text { Unilateral versus } \\
\text { bilateral balloon } \\
\text { kyphoplasty }\end{array}$} & \multirow[t]{2}{*}{6} & $\begin{array}{l}\text { Discordant } \\
\text { Review } \\
\text { authors }\end{array}$ & Step I & $\begin{array}{l}\text { Sun et al } \\
2016\end{array}$ & $\begin{array}{l}\text { AMSTAR } \\
\text { (Moderate quality) }\end{array}$ & $\begin{array}{l}\text { Non- } \\
\text { Cochrane }\end{array}$ & Low risk \\
\hline & & & & Our Choice & Step I & Feng 2015 & (Moderate quality) & $\begin{array}{l}\text { Non- } \\
\text { Cochrane }\end{array}$ & Low risk \\
\hline \multirow[t]{2}{*}{ Fu 2019} & \multirow[t]{2}{*}{ Function } & \multirow[t]{2}{*}{$\begin{array}{l}\text { Surgical versus } \\
\text { non-surgical } \\
\text { treatment }\end{array}$} & \multirow[t]{2}{*}{4} & $\begin{array}{l}\text { Discordant } \\
\text { Review } \\
\text { authors }\end{array}$ & Step H & $\begin{array}{l}\text { Rabi et al } \\
2015\end{array}$ & $\begin{array}{l}\text { AMSTAR } \\
\text { (High quality) }\end{array}$ & $\begin{array}{l}\text { Non- } \\
\text { Cochrane }\end{array}$ & High risk \\
\hline & & & & Our Choice & Step H & $\begin{array}{l}\text { Handoll } \\
2012\end{array}$ & (Highest quality) & Cochrane & Low risk \\
\hline \multirow[t]{2}{*}{ Zhao 2015b } & \multirow[t]{2}{*}{ Non-union } & \multirow[t]{2}{*}{ Plate fixation } & \multirow[t]{2}{*}{4} & $\begin{array}{l}\text { Discordant } \\
\text { Review } \\
\text { authors }\end{array}$ & Step I & $\begin{array}{l}\text { Ouyang } \\
2013\end{array}$ & $\begin{array}{l}\text { AMSTAR } \\
\text { (Moderate quality) }\end{array}$ & $\begin{array}{l}\text { Non- } \\
\text { Cochrane }\end{array}$ & Low risk \\
\hline & & & & Our Choice & Step H & $\begin{array}{l}\text { Heineman } \\
2010\end{array}$ & (Moderate quality) & $\begin{array}{l}\text { Non- } \\
\text { Cochrane }\end{array}$ & Low risk \\
\hline
\end{tabular}

\subsubsection{Frequency of agreement and disagreement in systematic review findings}

Ten out of $13(77 \%)$ discordant Jadad assessments led to agreement in the findings (direction of effect was the same) (Table 3$)$. The remaining three independent Jadad assessments that disagreed led to a different direction of the effect estimates.

Overall, 18 out of the 21 (86\%) independent Jadad assessments agreed in direction of the findings despite 13 having chosen a different SR. We present a case study in Appendix E to illustrate a case study of the clinical impact of choosing one SR using the Jadad algorithm.

\subsubsection{Cochrane versus non-Cochrane reviews chosen}

Of the 21 Jadad assessments, four Cochrane reviews were chosen either by us or the Discordant Review authors (Table 3). On four occasions we chose a Cochrane review, and twice the Discordant Review authors chose a Cochrane review. 
Table 3

Agreement and disagreement in systematic review findings from replicated Jadad assessments

\begin{tabular}{|c|c|c|c|c|c|c|c|c|c|}
\hline $\begin{array}{l}\text { Discordant } \\
\text { Review }\end{array}$ & $\begin{array}{l}\text { Discordant } \\
\text { Review authors' } \\
\text { or our Jadad } \\
\text { assessments }\end{array}$ & $\begin{array}{l}\text { Review(s) } \\
\text { chosen by the } \\
\text { Discordant } \\
\text { Review } \\
\text { (First author } \\
\text { Year) }\end{array}$ & $\begin{array}{l}\text { Cochrane } \\
\text { or non- } \\
\text { Cochrane } \\
\text { SR }\end{array}$ & $\begin{array}{l}\text { Risk of } \\
\text { bias in } \\
\text { the } \\
\text { review }\end{array}$ & $\begin{array}{l}\text { Type of } \\
\text { effect } \\
\text { estimate }\end{array}$ & $\begin{array}{l}\text { Pooled } \\
\text { effect } \\
\text { sizes } \\
\text { and } 95 \% \\
\mathrm{Cl}\end{array}$ & $\begin{array}{l}\text { Statistical } \\
\text { significance ( } p \text { - } \\
\text { value of the } \\
\text { effect estimate) }\end{array}$ & $\begin{array}{l}\text { Results } \\
\text { favourable, } \\
\text { null, or } \\
\text { unfavourable }\end{array}$ & $\begin{array}{l}\text { Direction } \\
\text { of effect }\end{array}$ \\
\hline \multirow[t]{2}{*}{$\begin{array}{l}\text { Bakdach } \\
2020\end{array}$} & $\begin{array}{l}\text { Discordant } \\
\text { Review authors }\end{array}$ & $\begin{array}{l}\text { Sardana } \\
2018\end{array}$ & $\begin{array}{l}\text { Non- } \\
\text { Cochrane }\end{array}$ & Low & Risk ratio & $\begin{array}{l}0.39 \\
(0.26- \\
0.59)\end{array}$ & 0.005 & Favourable & \multirow[t]{2}{*}{ Disagree } \\
\hline & Our Choice & $\begin{array}{l}\text { Tasios } 2019 \\
\text { (and Sardana } \\
\text { 2019) }\end{array}$ & $\begin{array}{l}\text { Non- } \\
\text { Cochrane }\end{array}$ & Low & Risk ratio & $\begin{array}{l}0.46 \\
(0.18- \\
1.15)\end{array}$ & 0.1 & Null & \\
\hline \multirow[t]{2}{*}{$\begin{array}{l}\text { Chalmers } \\
2015\end{array}$} & $\begin{array}{l}\text { Discordant } \\
\text { Review authors }\end{array}$ & $\begin{array}{l}\text { Pulavarti } \\
2007\end{array}$ & Cochrane & Low & Risk ratio & $\begin{array}{l}0.89 \\
(0.09 \\
8.72)\end{array}$ & 0.92 & Null & \multirow[t]{2}{*}{ Agree } \\
\hline & Our Choice & $\begin{array}{l}\text { Lenters } \\
\text { (and Pulavarti } \\
2007 \text { ) }\end{array}$ & $\begin{array}{l}\text { Non- } \\
\text { Cochrane }\end{array}$ & Low & Risk ratio & $\begin{array}{l}1.31 \\
(0.51, \\
3.34)\end{array}$ & 0.58 & Null & \\
\hline \multirow[t]{2}{*}{ Chen 2019} & $\begin{array}{l}\text { Discordant } \\
\text { Review authors }\end{array}$ & Shen 2017 & $\begin{array}{l}\text { Non- } \\
\text { Cochrane }\end{array}$ & Low & $\begin{array}{l}\text { Mean } \\
\text { difference }\end{array}$ & $\begin{array}{l}-17.39 \\
(-22.32 \\
-12.46)\end{array}$ & $<0.00001$ & Favourable & \multirow[t]{2}{*}{ Agree } \\
\hline & Our Choice & Dai et al 2017 & $\begin{array}{l}\text { Non- } \\
\text { Cochrane }\end{array}$ & Low & $\begin{array}{l}\text { Mean } \\
\text { difference }\end{array}$ & $\begin{array}{l}-2.83 \\
(-4.26 \\
-1.39)\end{array}$ & 0.0001 & Favourable & \\
\hline \multirow[t]{2}{*}{ Fu 2019} & $\begin{array}{l}\text { Discordant } \\
\text { Review authors }\end{array}$ & $\begin{array}{l}\text { Rabi et al } \\
2015\end{array}$ & $\begin{array}{l}\text { Non- } \\
\text { Cochrane }\end{array}$ & High & $\begin{array}{l}\text { Mean } \\
\text { difference }\end{array}$ & $\begin{array}{l}1.63 \\
(-2.84 \\
6.11)\end{array}$ & 0.47 & Null & \multirow[t]{2}{*}{ Agree } \\
\hline & Our Choice & Handoll 2012 & Cochrane & Low & $\begin{array}{l}\text { Mean } \\
\text { difference }\end{array}$ & $\begin{array}{l}2.36 \\
(-3.52 \\
8.24)\end{array}$ & 0.43 & Null & \\
\hline \multirow[t]{2}{*}{ Guo 2018} & $\begin{array}{l}\text { Discordant } \\
\text { Review authors }\end{array}$ & Xia 2014 & $\begin{array}{l}\text { Non- } \\
\text { Cochrane }\end{array}$ & High & Risk ratio & $\begin{array}{l}1.19 \\
(1.05, \\
1.35)\end{array}$ & 0.006 & Favourable & \multirow[t]{2}{*}{ Agree } \\
\hline & Our Choice & Hu 2011 & $\begin{array}{l}\text { Non- } \\
\text { Cochrane }\end{array}$ & Low & Odds Ratio & $\begin{array}{l}3.34 \\
(1.92, \\
5.81)\end{array}$ & $<0.0001$ & Favourable & \\
\hline \multirow[t]{2}{*}{ Houck 2017} & $\begin{array}{l}\text { Discordant } \\
\text { Review authors }\end{array}$ & Riboh 2014 & $\begin{array}{l}\text { Non- } \\
\text { Cochrane }\end{array}$ & Low & $\begin{array}{l}\text { Mean } \\
\text { difference }\end{array}$ & $\begin{array}{l}14.70 \\
(5.52 \\
23.87)\end{array}$ & 0.002 & Favourable & \multirow[t]{2}{*}{ Agree } \\
\hline & Our Choice & Chan 2014 & $\begin{array}{l}\text { Non- } \\
\text { Cochrane }\end{array}$ & High & $\begin{array}{l}\text { Mean } \\
\text { difference }\end{array}$ & $\begin{array}{l}1.05 \\
(0.03, \\
2.06)\end{array}$ & 0.04 & Favourable & \\
\hline \multirow[t]{2}{*}{$\begin{array}{l}\text { Mascarenhas } \\
2014\end{array}$} & $\begin{array}{l}\text { Discordant } \\
\text { Review authors }\end{array}$ & Millett 2014 & $\begin{array}{l}\text { Non- } \\
\text { Cochrane }\end{array}$ & Low & $\begin{array}{l}\text { Mean } \\
\text { difference }\end{array}$ & $\begin{array}{l}-3.7 \\
(-8.8 \\
1.4)\end{array}$ & 0.16 & Null & \multirow[t]{2}{*}{ Agree } \\
\hline & Our Choice & $\begin{array}{l}\text { Sheibani-Rad } \\
2013\end{array}$ & $\begin{array}{l}\text { Non- } \\
\text { Cochrane }\end{array}$ & High & $\begin{array}{l}\text { Mean } \\
\text { difference }\end{array}$ & $\begin{array}{l}0.159 \\
(-0.08, \\
0.40)\end{array}$ & 0.255 & Null & \\
\hline \multirow[t]{2}{*}{ Pekala 2019} & $\begin{array}{l}\text { Discordant } \\
\text { Review authors }\end{array}$ & Pabalan 2016 & $\begin{array}{l}\text { Non- } \\
\text { Cochrane }\end{array}$ & High & Odds ratio & $\begin{array}{l}0.99 \\
(0.75 \\
1.31)\end{array}$ & 0.95 & Null & \multirow[t]{2}{*}{ Agree } \\
\hline & Our Choice & $\begin{array}{l}\text { Nong } 2016 \\
\text { (and Pabalan } \\
\text { 2016) }\end{array}$ & $\begin{array}{l}\text { Non- } \\
\text { Cochrane }\end{array}$ & Low & Odds ratio & $\begin{array}{l}1.13 \\
(0.76- \\
1.69)\end{array}$ & 0.55 & Null & \\
\hline \multirow[t]{3}{*}{ Song 2016} & $\begin{array}{l}\text { Discordant } \\
\text { Review authors }\end{array}$ & Cao 2015 & $\begin{array}{l}\text { Non- } \\
\text { Cochrane }\end{array}$ & High & Risk ratio & $\begin{array}{l}0.41 \\
(0.07 \\
2.52)\end{array}$ & 0.34 & Null & \multirow[t]{3}{*}{ Agree } \\
\hline & $\begin{array}{l}\text { Discordant } \\
\text { Review authors }\end{array}$ & Wu 2015 & $\begin{array}{l}\text { Non- } \\
\text { Cochrane }\end{array}$ & Low & Risk ratio & $\begin{array}{l}0.98 \\
(0.2, \\
4.75)\end{array}$ & 0.98 & Null & \\
\hline & Our Choice & $\begin{array}{l}\text { Gurusamy } \\
2013\end{array}$ & Cochrane & Low & Odds ratio & $\begin{array}{l}0.49 \\
(0.05 \\
4.72)\end{array}$ & 0.54 & Null & \\
\hline
\end{tabular}




\begin{tabular}{|c|c|c|c|c|c|c|c|c|c|}
\hline $\begin{array}{l}\text { Discordant } \\
\text { Review }\end{array}$ & $\begin{array}{l}\text { Discordant } \\
\text { Review authors' } \\
\text { or our Jadad } \\
\text { assessments }\end{array}$ & $\begin{array}{l}\text { Review(s) } \\
\text { chosen by the } \\
\text { Discordant } \\
\text { Review } \\
\text { (First author } \\
\text { Year) }\end{array}$ & $\begin{array}{l}\text { Cochrane } \\
\text { or non- } \\
\text { Cochrane } \\
\text { SR }\end{array}$ & $\begin{array}{l}\text { Risk of } \\
\text { bias in } \\
\text { the } \\
\text { review }\end{array}$ & $\begin{array}{l}\text { Type of } \\
\text { effect } \\
\text { estimate }\end{array}$ & $\begin{array}{l}\text { Pooled } \\
\text { effect } \\
\text { sizes } \\
\text { and } 95 \% \\
\text { Cl }\end{array}$ & $\begin{array}{l}\text { Statistical } \\
\text { significance ( } p \text { - } \\
\text { value of the } \\
\text { effect estimate) }\end{array}$ & $\begin{array}{l}\text { Results } \\
\text { favourable, } \\
\text { null, or } \\
\text { unfavourable }\end{array}$ & $\begin{array}{l}\text { Direction } \\
\text { of effect }\end{array}$ \\
\hline \multirow[t]{2}{*}{ Tan 2018} & $\begin{array}{l}\text { Discordant } \\
\text { Review authors }\end{array}$ & Feng 2015 & $\begin{array}{l}\text { Non- } \\
\text { Cochrane }\end{array}$ & Low & $\begin{array}{l}\text { Mean } \\
\text { difference }\end{array}$ & $\begin{array}{l}-0.18 \\
(-0.36 \\
-0.00)\end{array}$ & 0.04 & Favourable & Disagree \\
\hline & Our Choice & Lin 2013 & $\begin{array}{l}\text { Non- } \\
\text { Cochrane }\end{array}$ & High & $\begin{array}{l}\text { Mean } \\
\text { difference }\end{array}$ & $\begin{array}{l}0.05 \\
(-0.49 \\
0.59)\end{array}$ & 0.87 & Null & \\
\hline \multirow[t]{2}{*}{ Xing 2016} & $\begin{array}{l}\text { Discordant } \\
\text { Review authors }\end{array}$ & Bellamy 2006 & Cochrane & High & $\begin{array}{l}\text { Mean } \\
\text { difference }\end{array}$ & $\begin{array}{l}-13.00 \\
(-17.77 \\
-8.33)\end{array}$ & $<0.00001$ & Favourable & Agree \\
\hline & Our Choice & $\begin{array}{l}\text { Richette } 2015 \\
\text { (and Bellamy } \\
\text { 2006) }\end{array}$ & $\begin{array}{l}\text { Non- } \\
\text { Cochrane }\end{array}$ & High & $\begin{array}{l}\text { Standardised } \\
\text { mean } \\
\text { difference }\end{array}$ & $\begin{array}{l}-0.21 \\
(-0.32 \\
-0.1)\end{array}$ & NR & Favourable & \\
\hline \multirow[t]{2}{*}{ Zhao 2015a } & $\begin{array}{l}\text { Discordant } \\
\text { Review authors }\end{array}$ & Ouyang 2013 & $\begin{array}{l}\text { Non- } \\
\text { Cochrane }\end{array}$ & Low & Risk ratio & $\begin{array}{l}1.20 \\
(0.63 \\
2.28)\end{array}$ & 0.58 & Null & Agree \\
\hline & Our Choice & $\begin{array}{l}\text { Heineman } \\
2010\end{array}$ & $\begin{array}{l}\text { Non- } \\
\text { Cochrane }\end{array}$ & Low & Risk Ratio & $\begin{array}{l}0.71 \\
(0.28 \\
1.76)\end{array}$ & 0.45 & Null & \\
\hline \multirow[t]{2}{*}{$\begin{array}{l}\text { Zhiyong } \\
2019\end{array}$} & $\begin{array}{l}\text { Discordant } \\
\text { Review authors }\end{array}$ & $\begin{array}{l}\text { Sun et al } \\
2016\end{array}$ & $\begin{array}{l}\text { Non- } \\
\text { Cochrane }\end{array}$ & Low & $\begin{array}{l}\text { Mean } \\
\text { difference }\end{array}$ & $\begin{array}{l}-0.12 \\
(-0.33 \\
0.09)\end{array}$ & 0.28 & Null & Disagree \\
\hline & Our Choice & Feng 2015 & $\begin{array}{l}\text { Non- } \\
\text { Cochrane }\end{array}$ & Low & $\begin{array}{l}\text { Mean } \\
\text { difference }\end{array}$ & $\begin{array}{l}-0.18 \\
(-0.36 \\
-0.00)\end{array}$ & 0.04 & Favourable & \\
\hline
\end{tabular}

\subsubsection{Interpretation of Jadad steps by the Discordant Review author}

Overall, there were major differences in the interpretation of the Jadad algorithm across Discordant Review author groups. Several Discordant Reviews $(n=12)$ did not implement the Jadad algorithm in sequential steps but rather, used components of the algorithm to assess SRs. In addition, various factors outside the Jadad algorithm were assessed by half $(n=10 / 21)$ Discordant Review authors, including: databases that were searched, the GRADE (Grading of Recommendations, Assessment, Development and Evaluations) approach, randomization method, methods used to measure outcomes, measures used to explore heterogeneity, measures used for establishing comparative superiority or inferiority, statistical approaches used in analyses, presence of subgroup analyses, software used to perform the analyses, and sources funding.

Most Discordant Reviews ( $n=12$ ) used Step I as the final decision step of the algorithm. We interpreted Step I to consist of (I1) publication status, (I2) quality of RCTs, (I3) language restrictions, and (I4) analysis of individual patient data (IPD). We operationalised publication status to be whether SRs included both published and unpublished (grey literature). In contrast, several Discordant Review authors interpreted this step to consider the date or recency of the review ( $\mathrm{n}$ $=5)$ or to account for only published literature $(n=3)$.

The second most common final decision step was Step H $(n=5)$. We interpreted Step H to consist of $(H 1)$ search strategies across SRs and (H2) inclusion criteria and duplicate independent screening of RCTs. Different Interpretations of H1 included: whether the SR was published in a medical journal, and which electronic databases were searched. Interpretations of $\mathrm{H} 2$ included: whether the SR reported publication status and language. Many Discordant Review authors were not clear in the details of how they interpreted this step. Some Discordant Review authors reported Step H as the final decision step but ignored the criteria for Step $G$ and selected the SR with the highest number of RCTs $(n=2)$. The rationale behind this decision was not reported.

\subsubsection{Time and ease of use in completing Steps $\mathrm{H}$ and $\mathrm{I}$ of the Jadad assessments}

\subsubsection{Time to do Steps $\mathrm{H}$ and I of the Jadad algorithm}

Of the 18 Jadad algorithm assessments completed for Steps $\mathrm{H}$ and I, the average time was 60 minutes per review (Appendix F and G, Table 1). Ten Jadad assessments took between 15 minutes and 47.5 minutes with an average of 4.3 SRs to assess (range 3-6). Nine out of the ten were rated as easy to assess, and one was rated as being moderately difficult to assess. These nine easy-rated assessments had evaluated Step $\mathrm{H}$ of the Jadad algorithm as the final decision step. The moderately rated assessment had evaluated Step I as the final decision step.

\subsubsection{Ease of use rating for final decision Steps $\mathrm{H}$ and $\mathrm{I}$ of the Jadad algorithm}

Of the 18 Jadad assessments we completed with final decision for Steps $\mathrm{H}$ and Step I, the median ease rating was easy (Appendix F and G, Table 1). Ten out of $18(56 \%)$ assessments were rated easy, six (33\%) were rated moderate, one moderate/hard (6\%), and one hard (6\%). All the assessments rated as easy were based on completing Step H. Of the easy assessments, the average number of SRs to assess was 4.4 (range 3-6 SRs). Of the eight moderate to hard assessments, three required assessment of Step I, and five Step H. They averaged 5.5 SRs to assess (range 2-10 SRs). By observation, we noticed that an 
easy Jadad assessment involved good reporting by the Discordant Review authors and the SR authors, the step assessed, and whether the Discordant Review interpreted the Jadad algorithm in a similar way than to us. By observation, we noticed that moderate to hard assessments involved inadequate reporting by the Discordant Review authors and discrepant data reported in their included SRs, having to conduct ROBIS assessments as the Discordant Review did not assess the quality of the SRs, and a greater number of SRs included.

\subsubsection{Comprehensiveness of the Jadad algorithm (gaps or completeness)}

We identified several missing methods for explaining discordance. The algorithm did not account for the date of last literature search, nor did it account for publication recency. The number of primary studies included in the SRs was also not considered within the Jadad algorithm. Lastly, the certainty of evidence, as measured by the GRADE or other approaches, was not examined by the algorithm.

\subsection{ROBIS assessments}

We assessed 98 SRs which were included in our 21 Discordant Reviews using the ROBIS tool. A total of 41 SRs were at low risk of bias, and 57 SRs were at high risk of bias. From the 21 Discordant Reviews, we chose 19 low risk SRs and 9 high risk SRs, while the authors chose 17 low risk SRs and 8 high risk SRs. A more detailed assessment as well as the full ROBIS assessments for each SR are found in Appendix H. Our ROBIS judgments of high or low risk of bias for each SR are found in Table 2.

\subsection{Discussion}

\subsection{Summary and interpretation of the most important results}

In our investigation, we identified research examining discordance across comparable SRs using the Jadad algorithm [8] and attempted to replicate their findings. In $62 \%$ of cases, we were unable to replicate the findings and ultimately chose a different "best evidence" SR. The lack of guidance on how to operationalise the Jadad algorithm likely contributed to the different interpretations, and ultimately disagreement between our choice and the Discordant Review authors' choice of SR. Several Discordant Reviews did not implement the Jadad algorithm in sequential steps which also may have led to us choosing a different review using the Jadad algorithm. B y observation, whenever a Cochrane SR was included in a Discordant Review, the authors or us chose the Cochrane review as the best evidence. Overall, the raters assessed the Jadad algorithm as easy to use, taking average time was 60 minutes with an average of 4.3 SRs to partially assess. By observation, we noticed that a Jadad assessments took a longer time when there was: (a) greater number of SRs to assess, (b) having to do a quality assessment for the included SRs (as this was missing from the Discordant Review), and (c) inadequate reporting by the Discordant Review authors.

Due to limited reporting, it was challenging to replicate or obtain a comprehensive understanding of Discordant Review authors' use of the Jadad algorithm. Inadequate reporting on how the authors interpreted the Jadad algorithm and operationalised each step led to challenges in extracting the required information. Often, Discordant Review authors only discussed the final decision step of the algorithm, and not the other steps taken that led to the final decision.

The Jadad algorithm has several limitations in terms of comprehensiveness. The algorithm does not account for the date of last literature search, publication recency, the number of RCTs included in the SRs and certainty of evidence assessment (e.g. using the GRADE approach). Moreover across 21 assessments, quality/risk of bias at the SRs was not assessed.

The exponential growth of SRs means duplication and redundant reviews will become a greater problem for clinicians and policy makers. As there is no similar tool to assess discordance, the Jadad algorithm will continue to be used by researchers given that there are no other alternatives, and is indeed still being used today [13-15]. However, the algorithm is out of date, and therefore fails to incorporate advances in biases, methodological and statistical approaches to evidence synthesis [45, 46]. Major methodological advances published after its emergence include the PRISMA reporting standard (2009 and updated in 2020; [47, 48]), AMSTAR (2007 and update in 2017; [22, 23]), ROBIS (2018; [25]), and GRADE (2009; [49]). Statistical advances of current interest include multiple imputation to model missing data, meta-regression and model selection, and network meta-analyses [45, 46], to name a few.

Intuitively, SR quality should be an important consideration when comparing reviews, but this was never considered by our team or the authors of the 21 included Discordant Reviews because the entire left side of the Jadad algorithm (Steps D, E and F) was never applied. This should be considered a design flaw in the algorithm. SR quality was only considered in the algorithm when SRs evaluated the same RCTs, which is highly unlikely with the exponential growth in research output [1] and the difficulty in locating trials. In our sample, it never happened. When two (or more) SRs asked clinical, public health, or policy questions with similar eligibility criteria it would be logical to evaluate SR quality using validated tools like AMSTAR-2 or risk of bias tools such as ROBIS and either choose the highest quality SR or eliminate lower quality SRs when there are several to choose from.

\subsection{Comparison of our study with other similar studies}

No methodological investigations or replications of the Jadad algorithm were identified, and therefore we are not aware of any study to which we could directly compare our study results. We are only aware of studies that applied the Jadad algorithm for choosing the best SR. This is surprising, as it is wellknown that in most cases there are several SRs available for a given question [3], and general rules for practitioners have been suggested on how to choose the best review [50]. Others have noted that unexplained discordance can also result in formulating the need to conduct a new review [11, 51]. This underpins the urgent need for further empirical investigations.

\subsection{Implications when clinicians encounter multiple discordant systematic reviews on the same question}

Page $16 / 22$ 
Evidence-based medicine is commonly defined as the conscientious, explicit, and judicious use of current best evidence in the process of decision-making related to patient care [52]. Medical knowledge grows every day, so that evidence is rapidly evolving, and it seems impossible to stay current [1]. For example, it is recommended that a general practitioner read 19 articles every day [53], and dedicate an average of one hour per week to keep abreast of the literature [53]. With the additional problem of conflicting results in seemingly identical research, clinicians may struggle to find the time and guidance on how to do this.

Without the help of an updated algorithm to assess discordant results across SRs, clinicians will have difficulty in identifying and choosing the best evidence and thus engaging in decision-making with their patients and clinical teams. Uncertainty, disagreements, and differences in SR results undermines the ability of a healthcare provider to make an informed clinical decision [29]. As an example of the clinical impact of discordant results of SRs [29], patient reimbursement for hyaluronic acid treatment was stopped because of some negative meta-analyses results [54, 55], despite the fact that other SRs [56, 57] cited beneficial effects, as did RCTs for certain preparations $[58,59]$.

In the meantime, we recommend that healthcare providers, policy makers, patients and researchers address conflicts between review findings by choosing the $\mathrm{SR}(\mathrm{s})$ with meta-analysis of RCTs that most: closely resembles their clinical, public health, or policy question; recent in terms of publication date; comprehensive in terms of number of included RCTs; and is at the lowest risk of bias.

\subsection{Strengths and limitations}

Our study has several strengths. First, we used consensus building strategies to develop clear instructions on how to operationalise the Jadad algorithm, and to ensure a consistent approach to assumptions and stepwise interpretation. We also adopted a systematic and transparent approach to address the objectives outlined in our protocol using SR guidance [20]. A comprehensive search strategy, including a search of the grey literature, was employed with no restrictions on language and publication status to minimise publication bias. To minimise error, screening, extractions, and assessments were completed by two independent reviewers, and subsequently compared. Any discrepancies were resolved upon consensus, and when necessary, with the involvement of a third reviewer. Pilot screening and pilot assessments were completed by reviewers and assessed to ensure consistency in understanding of the screening criteria, and definitions of coding and extracted items.

There are some aspects of our methods that should be considered limitations. Our assessments of discordance using the Jadad algorithm was conducted without clinical expertise on some conditions and interventions. We attempted to minimise the impact of this by including both methodologists and clinicians in our research team but given the breadth of topics addressed by these studies, judging the similarity and relevance of clinical, public health, or policy questions and eligibility criteria was at times difficult. It is also possible that a broader search of different databases may have identified more studies using the Jadad algorithm. This might indicate that the use of the Jadad algorithm might be more prevalent than our study indicates. Findings from this study are not directly generalisable to SRs that include both RCTs and non-RCTs, which would have greater sources of heterogeneity in their study results. Furthermore, our sample is mostly representative of orthopedic conditions and may lack generalisability.

\subsection{Future research}

A tool that has better agreement between decision makers, addresses all pertinent variables that may contribute to discordance, and is easier to implement is needed. Such a tool, whether it be a framework or stepwise algorithm, will need to be applicable to a variety of settings (i.e., SRs of primary studies with and without standard meta-analysis or network meta-analysis). The proposed tool would also need to incorporate recent methodological and statistical advances in evidence synthesis. Validation of such a tool could involve expert opinion obtained from consensus building methods (i.e., Delphi methods) and other methods proposed by Whiting [60] and Moher [61]. Reproducibility will also be important so inter-rater reliability would also be tested. Ideally, such a tool would not only assist the clinician, stakeholder, or researcher in choosing the most appropriate SR but guide the user in identifying the most likely sources of discordance.

To address the gap in investigations of discordance using algorithms or methods other than that of Jadad [8], we are currently analysing approximately 70 studies to identify how they assessed discordance. We are using a qualitative framework analysis to map out any stepwise approaches used. This study and the current one will inform the development of our new algorithm to assess discordance across SRs with similar clinical, public health, and policy questions.

Finally, a new tool to assess discordance should take into consideration the conclusiveness, or the stability of the results, of the SRs under question [62]. For example, as a first step in assessing redundant reviews, researchers should ascertain whether the question has reached saturation called "conclusiveness" [62-65]. For example, the Cochrane logo shows the summary results from a conclusive SR called "Antenatal corticosteroids for accelerating fetal lung maturation for women at risk of preterm birth" [66]. This review is conclusive, meaning it provides a definitive recommendation for an intervention based on at least one meta-analysis with a $p$-value $<0.05$, and has overall consensus among clinicians worldwide as to its effectiveness.

\subsection{Conclusions}

Our results suggest that the Jadad algorithm is not reliably reproducible between decision makers and is inadequate for several reasons. First, there is no comprehensive prescriptive guidance on how to apply the algorithm. Second, quality was not considered when assessing SRs, which represents a major design flaw in the algorithm. Third, this tool fails to incorporate advances in biases, methodological and statistical approaches to evidence synthesis.

In the absence of a tool to assess discordance across SRs, we recommend that clinicians, stakeholders, patients and researchers address conflicts between review findings by choosing the SR with meta-analysis of RCTs that most closely resemble their question, is the most recent (most recent search date), comprehensive (i.e. number of included RCTs), and is at the lowest risk of bias.

\subsection{Declarations}


Not applicable

\subsection{Consent for publication}

Not applicable.

\subsection{Availability of data and materials}

The data is freely available on the Open Science Framework. The final Jadad algorithm steps, our decision rules and supplementary results are found in the appendices, eight instructional videos are located at https://osf.io/2z7a5/, and our protocol is printed as a preprint on the Research Square server [20].

\subsection{Competing interests}

The authors declare that they have no competing interests.

\section{$5.5 \quad$ Funding}

No grants or funding were successful for this important study. Dr. Andrea Tricco currently holds a Tier 2 Canada Research Chair in Knowledge Synthesis. Dr. Carole Lunny is funded by a CIHR project grant for her postdoctoral research.

\subsection{Authors' contributions}

CL conceived of the study; all authors contributed to the design of the study and interpretation of Jadad algorithm steps; CL, NF, SW, LP, DW, BK, SS, ST, SK, WZ, DP, ER, HN, JZ, YC, AS, HN, RA, PS, KK screened studies;

CL, NF, SW, LP, DW, BK, WZ, ST, SS, SK, HN, JZ, YC, AS, HN, RA, PS, KK extracted data including characteristics of studies, Jadad assessments, and results data; CL, NF, SS, LP, DW, ST, NF, LP, DW, SW, JZ, YC, HN analysed the data; CL, NF, SK, SW, ACT, DP, ER, YC wrote the manuscript; all authors edited the manuscript; and all authors read and approved the final manuscript.

\subsection{Patient involvement}

Patients or the public were not involved in the design, or conduct, or reporting, or dissemination plans of our research

\subsection{Acknowledgements}

Not applicable.

\section{References}

1. Bornmann, L. and R. Mutz, Growth rates of modern science: A bibliometric analysis based on the number of publications and cited references. Journal of the Association for Information Science and Technology, 2015. 66(11): p. 2215-2222.

2. Bastian, H., P. Glasziou, and I. Chalmers, Seventy-five trials and eleven systematic reviews a day: how will we ever keep up? PLoS medicine, 2010. 7(9): p. e1000326.

3. Ioannidis, J.P., The mass production of redundant, misleading, and conflicted systematic reviews and meta-analyses. The Milbank Quarterly, 2016. 94(3): p. 485-514.

4. Taito, S., et al., Assessment of the Publication Trends of COVID-19 Systematic Reviews and Randomized Controlled Trials. Annals of Clinical Epidemiology, 2021. 3(2): p. 56-58.

5. Hoffmann, F., et al., Nearly 80 systematic reviews were published each day: Observational study on trends in epidemiology and reporting over the years 2000-2019. J Clin Epidemiol, 2021. 138: p. 1-11.

6. Bolland, M.J. and A. Grey, A case study of discordant overlapping meta-analyses: vitamin d supplements and fracture. PLoS One, 2014. 9(12): $\mathrm{p}$. e115934.

7. Daei, A., et al., Clinical information seeking behavior of physicians: A systematic review. International journal of medical informatics, 2020. 139: $\mathrm{p}$. 104144 .

8. Jadad, A.R., D.J. Cook, and G.P. Browman, A guide to interpreting discordant systematic reviews. Cmaj, 1997. 156(10): p. 1411-6.

9. Hartling, L., et al., A descriptive analysis of overviews of reviews published between 2000 and 2011. PLoS One, 2012. 7(11): p. e49667.

10. Lunny, C., et al., Toward a comprehensive evidence map of overview of systematic review methods: paper 1-purpose, eligibility, search and data extraction. Syst Rev, 2017. 6(1): p. 231.

11. Lunny, C., et al., Toward a comprehensive evidence map of overview of systematic review methods: paper 2-risk of bias assessment; synthesis, presentation and summary of the findings; and assessment of the certainty of the evidence. Syst Rev, 2018. 7(1): p. 159.

Page $18 / 22$ 
12. Pieper, D., et al., Overviews of reviews often have limited rigor: a systematic review. J Clin Epidemiol, 2012. 65(12): p. $1267-73$.

13. Bakdach, W.M.M. and R. Hadad, Effectiveness of different adjunctive interventions in the management of orthodontically induced white spot lesions: $A$ systematic review of systematic reviews and meta-analyses. Dental and Medical Problems, 2020.

14. Blom, A.W., et al., Common elective orthopaedic procedures and their clinical effectiveness: umbrella review of level 1 evidence. Bmj, 2021. 374: $\mathrm{p}$. n1511.

15. Chen, P., et al., Intra-articular platelet-rich plasma injection for knee osteoarthritis: a summary of meta-analyses. Journal of orthopaedic surgery and research, 2019. 14(1): p. 1-11.

16. Li, Q., et al., Minimally invasive versus open surgery for acute Achilles tendon rupture: a systematic review of overlapping meta-analyses. J Orthop Surg Res, 2016. 11(1): p. 65.

17. Mascarenhas, R., et al., Is double-row rotator cuff repair clinically superior to single-row rotator cuff repair: a systematic review of overlapping metaanalyses. Arthroscopy, 2014. 30(9): p. 1156-65.

18. Zhao, J.G., J. Wang, and L. Long, Surgical Versus Conservative Treatments for Displaced Midshaft Clavicular Fractures: A Systematic Review of Overlapping Meta-Analyses. Medicine (Baltimore), 2015. 94(26): p. e1057.

19. Higgins, J.P., Cochrane handbook for systematic reviews of interventions version 5.0. 1. The Cochrane Collaboration. http://www. cochrane-handbook. org, 2008.

20. Lunny C, T.S., Kanji S, Ferri N, Pieper D, Whitelaw S, Thabet P, Tasmin S, Nelson H, Reid E, Zhang JH., Identifying and addressing conflicting results across multiple discordant systematic reviews on the same topic: A protocol for a replication study of the Jadad algorithm [Internet]. 2021: Research Square. Available from: https://www.researchsquare.com/article/rs-931213/v1.

21. Lunny, C., et al., Bibliometric study of 'overviews of systematic reviews' of health interventions: evaluation of prevalence, citation and journal impact factor. Research Synthesis Methods, 2021.

22. Shea, B.J., et al., Development of AMSTAR: a measurement tool to assess the methodological quality of systematic reviews. BMC medical research methodology, 2007. 7(1): p. 1-7.

23. Shea, B.J., et al., AMSTAR 2: a critical appraisal tool for systematic reviews that include randomised or non-randomised studies of healthcare interventions, or both. bmj, 2017. 358.

24. Oxman, A.D. and G.H. Guyatt, Validation of an index of the quality of review articles. Journal of clinical epidemiology, 1991. 44(11): p. 1271-1278.

25. Whiting P, S.J., Churchill R. Introduction to ROBIS, a new tool to assess the risk of bias in a systematic review. in 23 rd Cochrane Colloquium. 2015. Vienna, Austria: John Wiley \& Sons.

26. Chalmers, P.N., et al., Do arthroscopic and open stabilization techniques restore equivalent stability to the shoulder in the setting of anterior glenohumeral instability? a systematic review of overlapping meta-analyses. Arthroscopy, 2015. 31(2): p. 355-63.

27. Campbell, J., N. Bellamy, and T. Gee, Differences between systematic reviews/meta-analyses of hyaluronic acid/hyaluronan/hylan in osteoarthritis of the knee. Osteoarthritis and cartilage, 2007. 15(12): p. 1424-1436.

28. Druyts, E., et al., Interpreting discordant indirect and multiple treatment comparison meta-analyses: an evaluation of direct acting antivirals for chronic hepatitis C infection. Clinical epidemiology, 2013. 5: p. 173.

29. Vavken, P. and R. Dorotka, A systematic review of conflicting meta-analyses in orthopaedic surgery. Clinical Orthopaedics and Related Research $\AA_{\text {, }}$ 2009. 467(10): p. 2723-2735.

30. Poolman, R.W., et al., Overlapping systematic reviews of anterior cruciate ligament reconstruction comparing hamstring autograft with bone-patellar tendon-bone autograft: why are they different? Journal of Bone \& Joint Surgery - American Volume, 2007. 89(7): p. $1542-52$.

31. Grassi, A., et al., Patellar resurfacing versus patellar retention in primary total knee arthroplasty: a systematic review of overlapping meta-analyses. Knee Surgery, Sports Traumatology, Arthroscopy, 2018. 26(11): p. 3206-3218.

32. Erickson, B.J., et al., Does operative treatment of first-time patellar dislocations lead to increased patellofemoral stability? A systematic review of overlapping meta-analyses. Arthroscopy: The Journal of Arthroscopic \& Related Surgery, 2015. 31(6): p. 1207-1215.

33. Xiaofeng Chen, M., et al., Is unilateral percutaneous kyphoplasty superior to bilateral percutaneous kyphoplasty for osteoporotic vertebral compression fractures? Evidence from a systematic review of discordant meta-analyses. Pain Physician, 2018. 21: p. 327-336.

34. Xu, B., et al., Is intramedullary fixation of displaced midshaft clavicle fracture superior to plate fixation? Evidence from a systematic review of discordant meta-analyses. International Journal of Surgery, 2017. 43: p. 155-162.

Page $19 / 22$ 
35. Song, G.M., et al., Laparoscopic cholecystectomy for acute cholecystitis: early or delayed?: Evidence from a systematic review of discordant metaanalyses. Medicine (Baltimore), 2016. 95(23): p. e3835.

36. Tan, G., et al., Unilateral versus bilateral percutaneous balloon kyphoplasty for osteoporotic vertebral compression fractures: A systematic review of overlapping meta-analyses. Medicine, 2018. 97(33).

37. Xing, D., et al., Intra-articular hyaluronic acid in treating knee osteoarthritis: a PRISMA-compliant systematic review of overlapping meta-analysis. Scientific reports, 2016. 6(1): p. 1-11.

38. Mascarenhas, R., et al., Does double-bundle anterior cruciate ligament reconstruction improve postoperative knee stability compared with single-bundle techniques? A systematic review of overlapping meta-analyses. Arthroscopy: The Journal of Arthroscopic \& Related Surgery, 2015. 31(6): p. 1185-1196.

39. Houck, D.A., et al., Early versus delayed motion after rotator cuff repair: a systematic review of overlapping meta-analyses. The American journal of sports medicine, 2017. 45(12): p. 2911-2915.

40. Pekala, P.A., et al., Fokl as a genetic factor of intervertebral disc degeneration: a PRISMA-compliant systematic review of overlapping meta-analyses. Journal of Clinical Neuroscience, 2019. 60: p. 36-43.

41. Cui Zhiyong, M., et al., Unilateral versus bilateral balloon kyphoplasty for osteoporotic vertebral compression fractures: a systematic review of overlapping meta-analyses. Pain physician, 2019. 22: p. 15-28.

42. Fu, B.s., et al., Surgical and Non-Surgical Treatment for 3-Part and 4-Part Fractures of the Proximal Humerus: A Systematic Review of Overlapping Meta-Analyses. Orthopaedic surgery, 2019. 11(3): p. 356-365.

43. Zhao, J.G., et al., Intramedullary nail versus plate fixation for humeral shaft fractures: a systematic review of overlapping meta-analyses. Medicine (Baltimore), 2015. 94(11): p. e599.

44. Guo, X.-w., et al., Shenyi Capsule ( $\mathbb{1}(\mathbb{Q})$ plus chemotherapy versus chemotherapy for non-small cell lung cancer: a systematic review of overlapping meta-analyses. Chinese journal of integrative medicine, 2018. 24(3): p. 227-231.

45. Gurevitch, J., et al., Meta-analysis and the science of research synthesis. Nature, 2018. 555(7695): p. 175-182.

46. Advances in Evidence Synthesis: special issue Cochrane Database of Systematic Reviews. Cochrane Database of Systematic Reviews, 2020. 9.

47. Moher, D., et al., Preferred reporting items for systematic reviews and meta-analyses: the PRISMA statement. PLoS medicine, 2009. 6(7): p. e1000097.

48. Page, M.J., et al., Updating guidance for reporting systematic reviews: development of the PRISMA 2020 statement. Journal of Clinical Epidemiology, 2021. 134: p. 103-112.

49. Brożek, J., et al., Grading quality of evidence and strength of recommendations in clinical practice guidelines: part 1 of 3. An overview of the GRADE approach and grading quality of evidence about interventions. Allergy, 2009. 64(5): p. 669-677.

50. BMJ. Multiple systematic reviews on the same question. https://bestpractice.bmj.com/info/us/toolkit/learn-ebm/multiple-systematic-reviews-on-thesame-question/. EBM Toolkit, Learn EBM, BMJ Best Practice 2021.

51. Robinson, K.A., et al., AHRQ Methods for Effective Health Care

Integrating Bodies of Evidence: Existing Systematic Reviews and Primary Studies, in Methods Guide for Effectiveness and Comparative Effectiveness Reviews. 2008, Agency for Healthcare Research and Quality (US): Rockville (MD).

52. Masic, I., M. Miokovic, and B. Muhamedagic, Evidence based medicine - new approaches and challenges. Acta informatica medica : AlM : journal of the Society for Medical Informatics of Bosnia \& Herzegovina : casopis Drustva za medicinsku informatiku BiH, 2008. 16(4): p. 219-225.

53. Mašić, I., Porodicna/obiteljska medicina: principi i praksa. 2007: na.

54. Arrich, J., et al., Intra-articular hyaluronic acid for the treatment of osteoarthritis of the knee: systematic review and meta-analysis. Cmaj, 2005. 172(8): p. $1039-1043$

55. Lo, G.H., et al., Intra-articular hyaluronic acid in treatment of knee osteoarthritis: a meta-analysis. Jama, 2003. 290(23): p. 3115-3121.

56. Bellamy, N., et al., Viscosupplementation for the treatment of osteoarthritis of the knee. Cochrane database of systematic reviews, 2006(2).

57. Wang, C.-T., et al., Therapeutic effects of hyaluronic acid on osteoarthritis of the knee: a meta-analysis of randomized controlled trials. JBJS, 2004. 86(3): p. 538-545.

58. Raynauld, J., et al., Effectiveness and safety of repeat courses of hylan GF 20 in patients with knee osteoarthritis. Osteoarthritis and cartilage, 2005. 13(2): p. 111-119. 
59. Raynauld, J.-P., et al., A prospective, randomized, pragmatic, health outcomes trial evaluating the incorporation of hylan GF 20 into the treatment paradigm for patients with knee osteoarthritis (Part 1 of 2): clinical results. Osteoarthritis and cartilage, 2002. 10(7): p. 506-517.

60. Whiting, P., et al., A proposed framework for developing quality assessment tools. Syst Rev, 2017. 6(1): p. 204.

61. Moher, D., et al., Guidance for developers of health research reporting guidelines. PLoS medicine, 2010. 7(2): p. e1000217.

62. Babić, A., et al., How to decide whether a systematic review is stable and not in need of updating: Analysis of Cochrane reviews. Research Synthesis Methods, 2020. 11(6): p. 884-890.

63. Cohen, S., et al., Conclusiveness of the Cochrane reviews in nutrition: a systematic analysis. European journal of clinical nutrition, 2014. 68(2): p. 143145.

64. Dosenovic, S., et al., Reasons and factors associated with inconclusiveness of systematic reviews about interventions for neuropathic pain. Journal of Comparative Effectiveness Research, 2020. 10(1): p. 67-75.

65. Mimouni, M., F. Mimouni, and F. Segev, Conclusiveness of the Cochrane eye and vision group reviews. BMC research notes, $2015.8(1)$ : p. 1-4.

66. Roberts, D., et al., Antenatal corticosteroids for accelerating fetal lung maturation for women at risk of preterm birth. Cochrane database of systematic reviews, 2017(3).

\section{Figures}

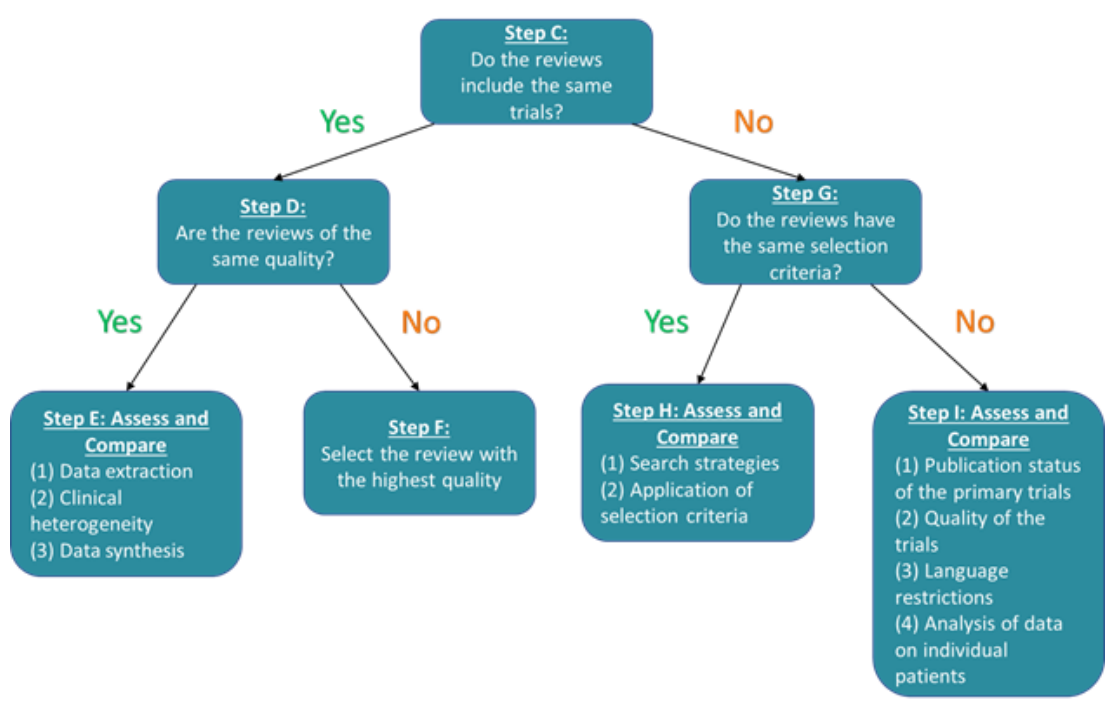

Figure 1

Jadad Algorithm (1997) 


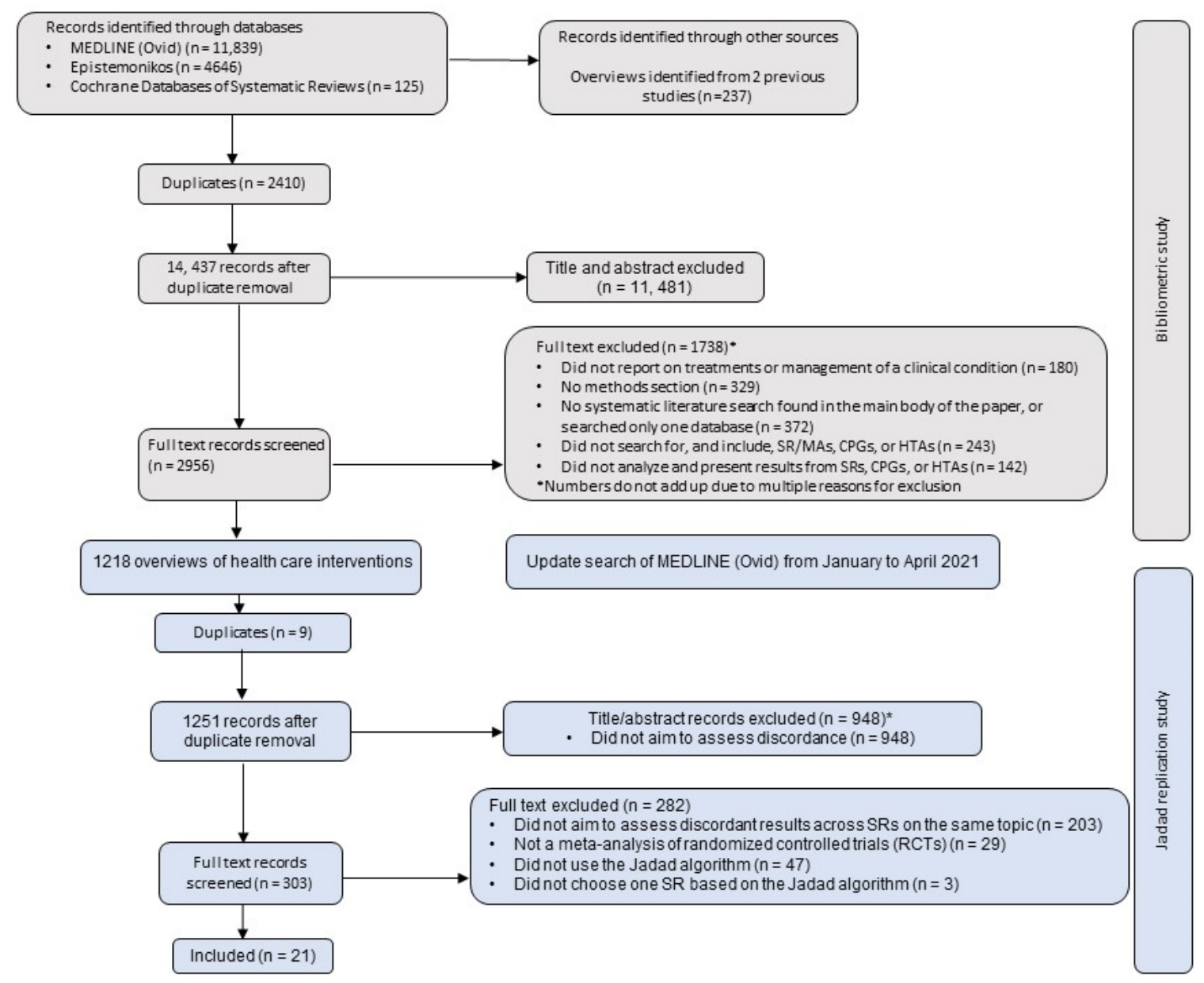

Figure 2

\section{Study selection flowchart}

Legend: The first section of the flowchart outlines the steps taken to select studies for the bibliometric study, and the section outlines our selection of studies for the Jadad replication

\section{Supplementary Files}

This is a list of supplementary files associated with this preprint. Click to download.

- AppendicesJadadreplicationstudy20220207.docx 